\title{
Direct Lactone Formation Using Hypervalent Iodine(III) Reagents with KBr via Selective C-H Abstraction Protocol
}

Toshifumi Dohi, Naoko Takenaga, Akihiro Goto, Akinobu Maruyama, and Yasuyuki Kita*

Graduate Scool of Pharmaceutical Sciences, Osaka University, 1-6 Yamada-oka, Suita, Osaka 565-0871, Japan

e-mail: kita@phs.osaka-u.ac.jp

\section{General Information}

${ }^{1} \mathrm{H}$ NMR and ${ }^{13} \mathrm{C}$ NMR spectra were recorded on a JEOL JMN-300 or EX-270 spectrometer in $\mathrm{CDCl}_{3}$ with tetramethylsilane as an internal standard. Data are reported as follows: chemical shift in ppm $(\delta)$, integration, multiplicity $(\mathrm{s}=$ singlet, $\mathrm{d}=\operatorname{doublet}, \mathrm{t}$ $=$ triplet, $\mathrm{q}=$ quartet, $\mathrm{br}=$ broad singlet, $\mathrm{m}=$ multiplet $)$, and coupling constant $(\mathrm{Hz})$. Infrared spectra (IR) were obtained on a Hitachi 270-50 spectrometer; absorptions are reported in reciprocal centimeters with the following relative intensities: $\mathrm{s}$ (strong), $\mathrm{m}$ (medium), or w (weak). Mass spectra were obtained on a Shimadzu GCMS-QP 5000 instrument with ionization voltages of $70 \mathrm{eV}$. High resolution mass spectra and Elemental analyses were performed by the Elemental Analysis Section of Osaka University. Column chromatography was performed with $\mathrm{SiO}_{2}$ (Merck Silica Gel 60 (230-400 mesh)).

\section{Materials}

p-Anisiodine diacetate $\mathbf{1}$ was obtained as a white powder from commercially available $p$-iodoanisole by the known method. ${ }^{1}$ Carboxylic acid $\mathbf{2} \mathbf{g}^{2}$ and $\mathbf{2 g}^{3}$ were prepared according to the literature procedure. Other starting materials were commercially 
available and used as received.

General Procedure for Direct Lactone Forming Reaction from Carboxylic Acids Using Aryliodine(III) diacetates 1 with $\mathrm{KBr}$ (Table 1)

In a flame-dried two-necked round bottomed flask, under nitrogen, to a stirred suspension of 4-pheyl butyric acid $2 \mathbf{2 a}(82 \mathrm{mg}, 0.5 \mathrm{mmol})$ and $\mathrm{KBr}(60 \mathrm{mg}, 0.5 \mathrm{mmol})$ in dry $\mathrm{CH}_{2} \mathrm{Cl}_{2}(5 \mathrm{~mL})$, the iodine(III) reagent $1(211 \mathrm{mg}, 0.6 \mathrm{mmol})$ was added and vigorously stirred for 8 hours at $30{ }^{\circ} \mathrm{C}$. After checking the reaction completion by TLC, saturated $\mathrm{NaHCO}_{3}$ aq. was added to the mixture, and then stirred for an additional 5 minutes. The organic layer was separated, washed with saturated $\mathrm{NaHCO}_{3}$ aq., dilute sodium thiosulfate aq. and dried over anhydrous $\mathrm{Na}_{2} \mathrm{SO}_{4}$. After removal of the solvents, the residue was subjected to silica-gel column chromatography (eluents: $n$-hexane/AcOEt) to give the $\gamma$-phenyl $\gamma$-butyrolactone 3a $(65 \mathrm{mg}, 80 \%)$ as a white powder. Unreacted $\mathbf{2 a}(9.8 \mathrm{mg}, 12 \%)$ was recovered from the combined aqueous phase by extraction of dichloromethane after acidifying the solution.

Dihydro-5-phenyl-2(3H)-furanone (3a): ${ }^{4}$ white powder; m.p. $35-36{ }^{\circ} \mathrm{C}, R_{f}=0.21$ (hexane/EtOAc $=3 / 1) ;{ }^{1} \mathrm{H}$ NMR $\left(300 \mathrm{MHz}, \mathrm{CDCl}_{3}\right): \delta$ 2.11-2.29 $(1 \mathrm{H}, \mathrm{m}), 2.56-2.74$ $(3 \mathrm{H}, \mathrm{m}), 5.51(1 \mathrm{H}, \mathrm{dd}, J=7.8,6.3 \mathrm{~Hz}), 7.25-7.68(5 \mathrm{H}, \mathrm{m}) ;{ }^{13} \mathrm{C} \mathrm{NMR}(75 \mathrm{MHz}$, $\left.\mathrm{CDCl}_{3}\right): \delta 28.91,30.92,81.18,125.23,128.40,128.73,139.33,176.87$; IR $(\mathrm{KBr}$, $\mathrm{cm}^{-1}$ ): 3034 m, 2930 m, 1767 s, 1606 w, 1497 m, 1455 m, 1417 m, 1329 m, 1286 m, 1215 s, 1176 s, 1141 s, 1080 m, 1021 s, 985 m, 940 s, 913 m, 890 m, 826 m, 757 s, 700 s, $678 \mathrm{~m}, 530 \mathrm{~m}$; MS, $m / z: 162\left(\mathrm{M}^{+}\right)$. 
Table . Direct Aryl Lactone Forming Reaction from Aliphatic Carboxylic Acids 2

(2arboxylic acid (2)

Dihydro-5-(4-methoxyphenyl)-2(3H)-furanone (3b): ${ }^{5}$ white powder; m.p. $53-54{ }^{\circ} \mathrm{C}$, $R_{f}=0.31($ hexane/EtOAc $=2 / 1) ;{ }^{1} \mathrm{H}$ NMR $\left(300 \mathrm{MHz}, \mathrm{CDCl}_{3}\right): \delta 2.13-2.24(1 \mathrm{H}, \mathrm{m})$, 2.57-2.70 (3H, m), $3.81(3 \mathrm{H}, \mathrm{s}), 5.43-5.49(1 \mathrm{H}, \mathrm{m}), 6.91(2 \mathrm{H}, \mathrm{d}, J=8.7 \mathrm{~Hz}), 7.26(2 \mathrm{H}$, $\mathrm{d}, J=8.7 \mathrm{~Hz}) ;{ }^{13} \mathrm{C} \mathrm{NMR}\left(75 \mathrm{MHz}, \mathrm{CDCl}_{3}\right): \delta 29.20,30.87,55.29,81.32,114.05$, 
126.92, 131.08, 159.69, 176.95; IR (KBr, cm $\left.{ }^{-1}\right): 3001$ m, 2914 m, 2837 m, 1769 s, 1614 s, 1587 m, 1514 s, 1418 m, 1250 s, 1217 s, 1142 s, 1111 m, 1032 s, 981 m, 937 m, 893 m, 808 m, 772 w, 669 m, 637 w, 530 m; MS, m/z: $192\left(\mathrm{M}^{+}\right)$.

Dihydro-5-(4-nitrophenyl) -2(3H)-furanone (3c): ${ }^{6}$ white powder; m.p. $226-228{ }^{\circ} \mathrm{C}, R_{f}$ $=0.33$ (hexane/EtOAc $=1 / 1) ;{ }^{1} \mathrm{H}$ NMR $\left(300 \mathrm{MHz}, \mathrm{CDCl}_{3}\right): \delta$ 2.09-2.25 $(1 \mathrm{H}, \mathrm{m})$, 2.63-2.85 (3H, m), 5.58-5.65 (1H, m), $7.53(2 \mathrm{H}, \mathrm{d}, J=8.7 \mathrm{~Hz}), 8.27(2 \mathrm{H}, \mathrm{d}, J=8.7 \mathrm{~Hz})$; ${ }^{13} \mathrm{C}$ NMR $\left(75 \mathrm{MHz}, \mathrm{CDCl}_{3}\right): \delta 28.60,30.87,79.60,124.07,125.91,146.56,147.80$, 176.00; IR (KBr, cm ${ }^{-1}$ ): 3080 w, 2928 w, 2855 w, 1788 s, 1607 m, 1520 s, 1456 w, 1416 m, 1350 s, 1292 m, 1263 m, 1215 m, 1175 s, 1142 s, 1109 m, 1032 s, 995 m, 941 m, 854 s, 818 m, 750 m, 700 m; MS, m/z: $207\left(\mathrm{M}^{+}\right)$.

5-(4-Fluorophenyl)-dihydro-2(3H)-furanone $\quad(\mathbf{3 d}):^{7} \quad$ colorless $\quad$ oil; $\quad R_{f}=0.14$ (hexane/EtOAc $=3 / 1) ;{ }^{1} \mathrm{H}$ NMR $\left(300 \mathrm{MHz}, \mathrm{CDCl}_{3}\right): \delta 2.12-2.27(1 \mathrm{H}, \mathrm{m}), 2.59-2.74$ $(3 \mathrm{H}, \mathrm{m}), 5.50(1 \mathrm{H}, \mathrm{dd}, J=8.3,6.0 \mathrm{~Hz}), 7.04-7.15(2 \mathrm{H}, \mathrm{m}), 7.28-7.36(2 \mathrm{H}, \mathrm{m}) ;{ }^{13} \mathrm{C} \mathrm{NMR}$ (75 MHz, $\left.\mathrm{CDCl}_{3}\right): \delta 29.01,31.06,80.65,115.60,115.88,127.13,127.24,176.63 ; \mathrm{IR}$ $\left(\mathrm{KBr}, \mathrm{cm}^{-1}\right): 3080$ w, 2926 w, 1778 s, 1609 m, 1514 s, 1458 m, 1421 m, 1329 m, 1227 s, 1177 s, 1142 s, 1099 w, 1022 m, 986 m, 941 m, 891 m, 837 m, 808 m, 783 w, 743 w, $530 \mathrm{w} ; \mathrm{MS}, m / z: 180\left(\mathrm{M}^{+}\right)$.

5-(4-Bromophenyl)-dihydro-2(3H)-furanone (3e): ${ }^{8}$ white powder; m.p. $81-82{ }^{\circ} \mathrm{C}, R_{f}=$ 0.35 (hexane/EtOAc $=2 / 1) ;{ }^{1} \mathrm{H}$ NMR $\left(300 \mathrm{MHz}, \mathrm{CDCl}_{3}\right): \delta 2.06-2.23(1 \mathrm{H}, \mathrm{m})$, 2.60-2.74 (3H, m), $5.47(1 \mathrm{H}, \mathrm{dd}, J=8.1,6.0 \mathrm{~Hz}), 7.22(2 \mathrm{H}, \mathrm{d}, J=8.4 \mathrm{~Hz}), 7.52(2 \mathrm{H}, \mathrm{d}$, $J=8.4 \mathrm{~Hz}) ;{ }^{13} \mathrm{C}$ NMR $\left(75 \mathrm{MHz}, \mathrm{CDCl}_{3}\right): \delta 28.82,30.89,80.41,122.35,126.92$, 131.88, 138.37, 176.53; IR (KBr,_cm $\left.{ }^{-1}\right): 3058$ w, 2949 m, 1769 s, 1593 m, 1493 s, 1456 m, 1416 m, 1327 m, 1279 m, 1213 s, 1142 s, 1011 s, 988 m, 937 s, 889 m, 806 s, 716 m, $669 \mathrm{~m}, 629 \mathrm{w}, 606 \mathrm{w}, 529 \mathrm{~m}$; MS, $m / z: 240\left(\mathrm{M}^{+}\right)$. 
5-[1,1'-Biphenyl]-4-yl-dihydro-2(3H)-furanone (3f): ${ }^{9}$ white powder; m.p. 105-106 ${ }^{\circ} \mathrm{C}$, $R_{f}=0.54($ hexane/EtOAc $=1 / 1) ;{ }^{1} \mathrm{H}$ NMR $\left(300 \mathrm{MHz}, \mathrm{CDCl}_{3}\right): \delta 2.15-2.33(1 \mathrm{H}, \mathrm{m})$, 2.63-2.76 (3H, m), $5.56(1 \mathrm{H}, \mathrm{t}, J=7.5 \mathrm{~Hz}), 7.25-7.45(5 \mathrm{H}, \mathrm{m}), 7.57-7.68(4 \mathrm{H}, \mathrm{m}) ;{ }^{13} \mathrm{C}$ NMR $\left(75 \mathrm{MHz}, \mathrm{CDCl}_{3}\right): \delta 28.93,30.91,81.01,125.74,127.05,127.43,127.52$, 128.80, 138.26, 140.36, 141.39, 176.82; IR (KBr, cm $\left.{ }^{-1}\right): 3041$ w, 2926 w, 1767 s, 1566 w, 1487 m, 1414 m, 1327 m, 1294 m, 1219 m, 1182 s, 1148 s, 1119 w, 1018 m, 980 m, $941 \mathrm{~s}, 895$ m, 843 m, 812 m, 725 m, 690 m, 642 w; MS, m/z: $238\left(\mathrm{M}^{+}\right)$.

Dihydro-5,5-diphenyl-2(3H)-furanone (3g): ${ }^{10}$ white powder; m.p. $88-89{ }^{\circ} \mathrm{C}, R_{f}=0.71$ (hexane/EtOAc $=1 / 1) ;{ }^{1} \mathrm{H}$ NMR $\left(300 \mathrm{MHz}, \mathrm{CDCl}_{3}\right): \delta$ 2.54-2.65 (2H, m), 2.87-2.99 $(2 \mathrm{H}, \mathrm{m}), 7.23-7.50(10 \mathrm{H}, \mathrm{m}) ;{ }^{13} \mathrm{C} \mathrm{NMR}\left(75 \mathrm{MHz}, \mathrm{CDCl}_{3}\right): \delta 29.00,35.62,89.67$, 125.34, 127.85, 128.56, 142.97, 176.06; IR (KBr, cm $\left.{ }^{-1}\right): 3060$ m, 3028 m, 3003 w, 2929 w, 1770 s, 1598 m, 1583 w, 1492 s, 1449 s, 1417 m, 1286 m, 1215 s, 1163 s, 1090 m, 1043 s, 981 s, 921 s, 903 s, 883 m, 846 w, 811 m, 780 w, 751 s, 738 s, 699 s, 663 m, 612 m, $561 \mathrm{w} ; \mathrm{MS}, m / z: 238\left(\mathrm{M}^{+}\right)$.

In the reaction of carboxylic acid $\mathbf{2 h}$, trans-lactone product $\mathbf{3 h}$ produced as a major product together with a regioisomeric cis-lactone under the reaction conditions. After the typical work-up, a mixture of crude products $\mathbf{3 h}$ and the cis-regioisomer was obtained by evaporation, and then it was subjected to ${ }^{1} \mathrm{H}$ NMR to determine a ratio of $\mathbf{3 h}$ and its cis-regioisomer. ${ }^{11}$ Integrations of the benzyl proton of $\mathbf{3 h}(\delta 4.9 \mathrm{ppm}, 1 \mathrm{H})$ and the cis-regioisomer $(\delta 5.5,1 \mathrm{H})$ indicated that these isomers presented in a ratio of ca. 100: 5 (Chart 1). Purification by column chromatography $\left(\mathrm{SiO}_{2} /\right.$ hexane-AcOEt) gave 3h containing $4 \%$ of the cis-regioisomer in $50 \%$ yield.

trans-Dihydro-4-methyl-5-phenyl-2(3H)-furanone $\quad(3 \mathrm{~h}, \quad$ containing $\quad 4 \% \quad$ of 
cis-regioisomer): ${ }^{11}$ white powder; $R_{f}=0.29$ (hexane/EtOAc $\left.=3 / 1\right) ;{ }^{1} \mathrm{H}$ NMR $(300 \mathrm{MHz}$, $\left.\mathrm{CDCl}_{3}\right): \delta 1.20(3 \mathrm{H}, \mathrm{d}, J=6.4 \mathrm{~Hz}), 2.28-2.55(2 \mathrm{H}, \mathrm{m}), 2.80(1 \mathrm{H}, \mathrm{dd}, J=16.8,7.5 \mathrm{~Hz})$, $4.94(1 \mathrm{H}, \mathrm{d}, J=8.4 \mathrm{~Hz}),, 7.22-7.56(5 \mathrm{H}, \mathrm{m}) ;{ }^{13} \mathrm{C} \mathrm{NMR}\left(75 \mathrm{MHz}, \mathrm{CDCl}_{3}\right): \delta 16.45$, 37.21, 39.83, 88.14, 125.89, 128.68 (x 2), 137.88, 176.13; IR (KBr, cm $\left.{ }^{-1}\right): 3034$ m, 2963 m, 2930 m, 2876 m, 1788 s, 1497 m, 1456 m, 1418 m, 1383 m, 1279 s, 1211 s, 1142 s, 1076 m, 1005 s, 947 m, 912 m, 891 w, 862 m, 802 m, 756 s, 700 s, 633 m, 598 w, 550 $\mathrm{w}$; MS, $m / z: 176\left(\mathrm{M}^{+}\right)$.

Tetrahydro-6-phenyl-2H-pyran-2-one (3i): ${ }^{12}$ white powder; m.p. $76-78{ }^{\circ} \mathrm{C}, R_{f}=0.21$ (hexane/EtOAc $=3 / 1) ;{ }^{1} \mathrm{H}$ NMR $\left(300 \mathrm{MHz}, \mathrm{CDCl}_{3}\right): \delta 1.82-2.04(3 \mathrm{H}, \mathrm{m}), 2.13-2.21$ $(1 \mathrm{H}, \mathrm{m}), 2.50-2.76(2 \mathrm{H}, \mathrm{m}), 5.35(1 \mathrm{H}, \mathrm{dd}, J=10.5,3.3 \mathrm{~Hz}), 7.25-7.42(5 \mathrm{H}, \mathrm{m}) ;{ }^{13} \mathrm{C}$ NMR (75 MHz, $\left.\mathrm{CDCl}_{3}\right): \delta 18.50,29.42,30.42,81.56,125.63,128.17,128.51,139.67$, 171.30; IR (KBr, cm $\left.{ }^{-1}\right): 3063$ m, 3033 m, 2955 m, 2881 m, 1738 s, 1605 w, 1495 m, 1455 s, 1444 m, 1330 s, 1238 s, 1187 s, 1158 s, 1043 s, 1002 m, 965 s, 934 s, 875 m, 822 w, 755 s, 700 s, 653 m, 625 m, 571 w, 533 m; MS, m/z: $176\left(\mathrm{M}^{+}\right)$.

7-Phenyl-2-oxepanone (3j): ${ }^{13}$ white powder; m.p. 66-67 ${ }^{\circ} \mathrm{C}, R_{f}=0.58$ (hexane/EtOAc $=1 / 1) ;{ }^{1} \mathrm{H}$ NMR $\left(300 \mathrm{MHz}, \mathrm{CDCl}_{3}\right): \delta 1.54-1.87(2 \mathrm{H}, \mathrm{m}), 1.96-2.33(4 \mathrm{H}, \mathrm{m}), 2.70-2.84$ $(2 \mathrm{H}, \mathrm{m}), 5.26-5.37(1 \mathrm{H}, \mathrm{m}), 7.24-7.49(5 \mathrm{H}, \mathrm{m}) ;{ }^{13} \mathrm{C} \mathrm{NMR}\left(75 \mathrm{MHz}, \mathrm{CDCl}_{3}\right): \delta 22.83$, $28.62,34.95,37.46,82.12,125.84,128.09,128.56,140.75,174.92$; IR $\left(\mathrm{KBr}, \mathrm{cm}^{-1}\right)$ : 3029 w, 2936 m, 2862 w, 1732 s, 1497 w, 1447 m, 1329 m, 1254 s, 1229 s, 1165 s, 1140 s, 1090 m, 1045 m, 1011 s, 897 w, 858 m, 752 m, 700 s, 584 m; MS, m/z: $190\left(\mathrm{M}^{+}\right)$. 


\section{Chart 1.}

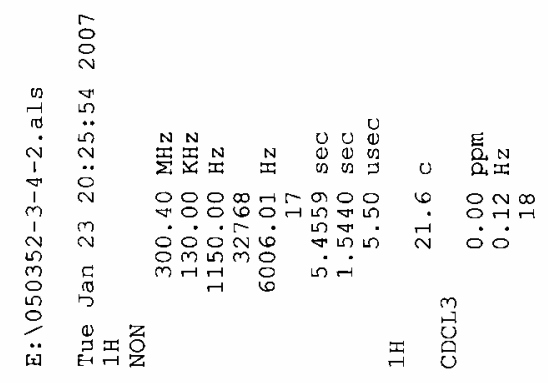

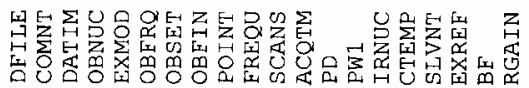

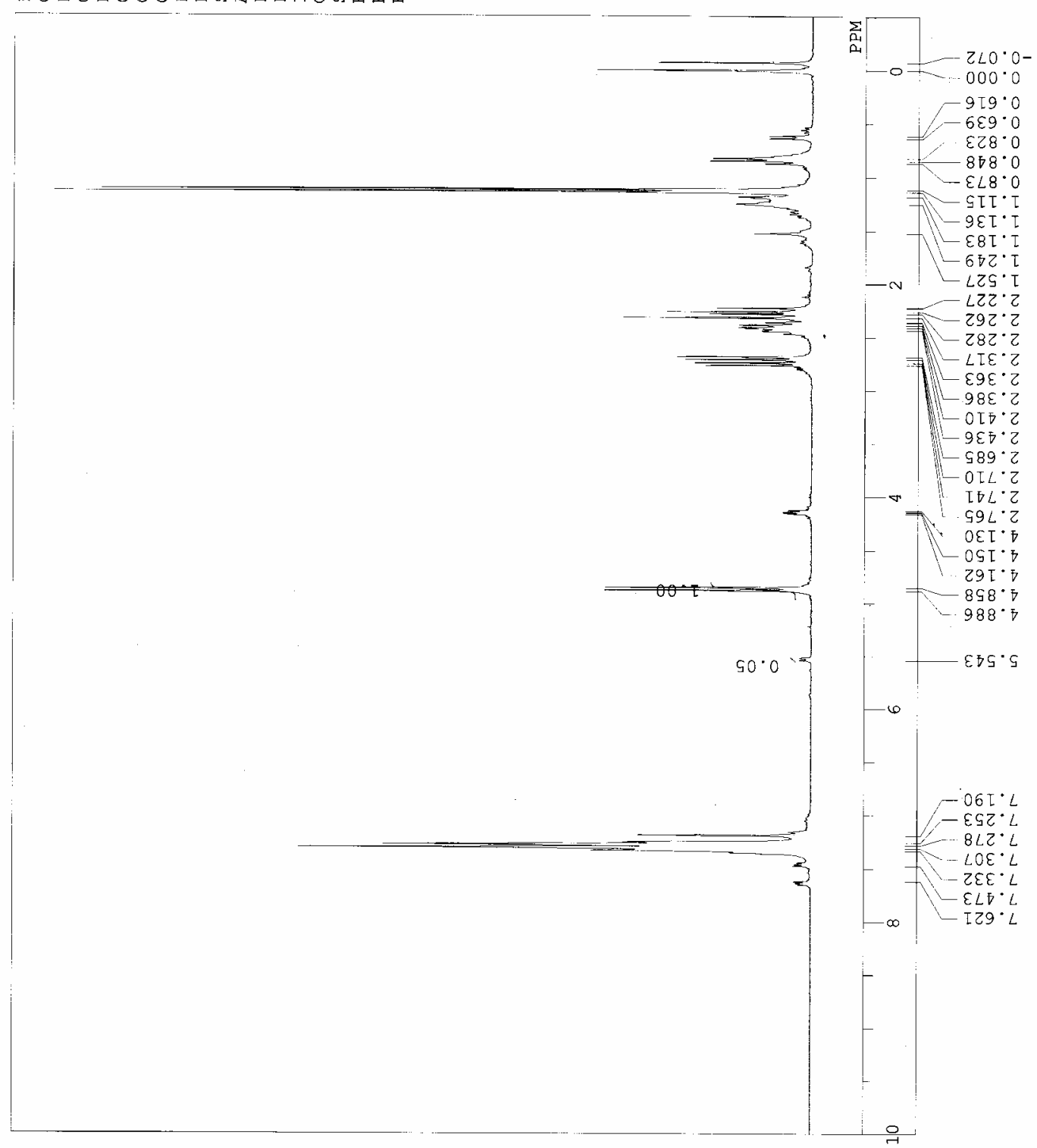




\section{General Procedure for Direct Lactone Forming Reaction from Benzoic Acids Using}

\section{$P I D A$ with $\mathrm{KBr}$}

In a flame-dried two-necked round bottomed flask, under nitrogen, to a stirred suspension of 2-ethyl benzoic acid (75 $\mathrm{mg}, 0.5 \mathrm{mmol})$ and $\mathrm{KBr}(60 \mathrm{mg}, 0.5 \mathrm{mmol})$ in dry $\mathrm{CH}_{2} \mathrm{Cl}_{2}(5 \mathrm{~mL})$, thePIDA (193 $\left.\mathrm{mg}, 0.6 \mathrm{mmol}\right)$ was added. The resulting mixture was stirred under $\mathrm{N}_{2}$ (balloon) at $40{ }^{\circ} \mathrm{C}$. for $8 \mathrm{~h}$. TLC check of the reaction mixture confirmed full conversion of the starting benzoic acid. After cooling, saturated $\mathrm{NaHCO}_{3}$ aq. was added to the mixture, and then stirred for an additional 5 minutes. Product $\mathbf{4 b}$ (60 mg, 81\%) was isolated as colorless oil from the organic layer by evaporation of the solvents, and successive silica-gel column chromatography (eluents: $n$-hexane/AcOEt).
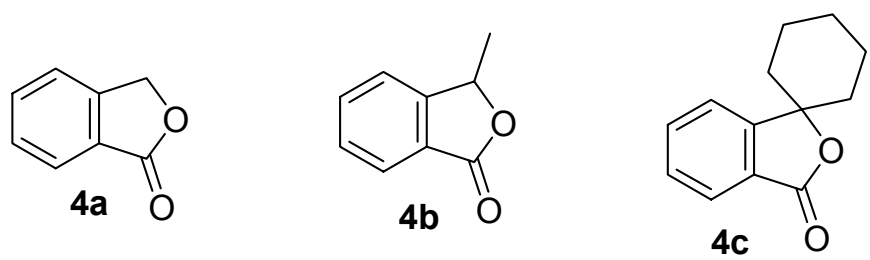

In the reaction of 2-alkyl benzoic acid leading to phthalides 4 , more reactive phenyliodine(III) diacetate (PIDA) usually gave better results than $p$-anisiodine diacetate $\mathbf{1}$. When using the reagent $\mathbf{1}$, large amount of starting materials were remained after 24 hours even at higher temperature $\left(50{ }^{\circ} \mathrm{C}\right)$.

3-Methylphthalide (4b): ${ }^{14}$ colorless oil; $R_{f}=0.30($ hexane/EtOAc $=4 / 1) ;{ }^{1} \mathrm{H}$ NMR $\left(300 \mathrm{MHz}, \mathrm{CDCl}_{3}\right): \delta 1.65(3 \mathrm{H}, \mathrm{d}, J=6.6 \mathrm{~Hz}), 5.58(1 \mathrm{H}, \mathrm{q}, J=6.6 \mathrm{~Hz}), 7.46(1 \mathrm{H}, \mathrm{d}, J=$ $7.8 \mathrm{~Hz}), 7.53(1 \mathrm{H}, \mathrm{t}, J=7.5 \mathrm{~Hz}), 7.66-7.73(1 \mathrm{H}, \mathrm{m}), 7.89(1 \mathrm{H}, \mathrm{d}, J=7.8 \mathrm{~Hz}) ;{ }^{13} \mathrm{C} \mathrm{NMR}$ $\left(75 \mathrm{MHz}, \mathrm{CDCl}_{3}\right): \delta 20.30,77.66,121.50,125.56,125.67,128.97,133.99,151.11$, 170.38 ; IR (KBr, cm $\left.{ }^{-1}\right): 3063$ w, 2982 m, 2932 m, 1759 s, 1614 m, 1597 m, 1468 m, 
$1454 \mathrm{~m}, 1377 \mathrm{~m}, 1346 \mathrm{~s}, 1286 \mathrm{~s}, 1215 \mathrm{~s}, 1196 \mathrm{~m}, 1155 \mathrm{~m}, 1105 \mathrm{~m}, 1080 \mathrm{~m}, 1043 \mathrm{~s}$, 1013 s, 959 m, 924 w, 887 w, 802 m, 764 s, 739 w, 696 s, 602 m, 573 m, 530 m; MS, $m / z: 148\left(\mathrm{M}^{+}\right)$.

Phthalide (4a): ${ }^{15}$ white powder; m.p. $73-74{ }^{\circ} \mathrm{C}, R_{f}=0.38($ hexane/EtOAc $=2 / 1) ;{ }^{1} \mathrm{H}$ NMR (300 MHz, $\left.\mathrm{CDCl}_{3}\right): \delta 5.34(2 \mathrm{H}, \mathrm{s}), 7.49-7.66(2 \mathrm{H}, \mathrm{m}), 7.68-7.76(1 \mathrm{H}, \mathrm{m}), 7.93$ $(1 \mathrm{H}, \mathrm{d}, J=7.5 \mathrm{~Hz}) ;{ }^{13} \mathrm{C} \mathrm{NMR}\left(75 \mathrm{MHz}, \mathrm{CDCl}_{3}\right): \delta 69.62,122.06,125.64,125.68$, 128.98, 133.97, 146.47, 171.08 ; IR (KBr, cm $\left.{ }^{-1}\right): 3061$ w, 2943 w, 2878 w, 1747 s, 1620 m, 1597 m, 1366 s, 1313 m, 1288 s, 1219 s, 1157 m, 1107 m, 1051 s, 1001 s, 974 s, 912 w, 858 w, 800 m, 739 s, 698 m, 681 m ,590 w, 565 w; MS, m/z: $134\left(\mathrm{M}^{+}\right)$.

Spiro[cyclohexane-1,1'(3'H)-isobenzofuran]-3'-one (4c): ${ }^{16}$ white powder; m.p. 77-78 ${ }^{\circ} \mathrm{C}, R_{f}=0.39($ hexane/EtOAc $=4 / 1) ;{ }^{1} \mathrm{H}$ NMR $\left(300 \mathrm{MHz}, \mathrm{CDCl}_{3}\right): \delta 1.60-2.10(10 \mathrm{H}$, m), $7.40(1 \mathrm{H}, \mathrm{d}, J=7.5 \mathrm{~Hz}), 7.50(1 \mathrm{H}, \mathrm{t}, J=7.2 \mathrm{~Hz}), 7.61-7.69(1 \mathrm{H}, \mathrm{m}), 7.87(1 \mathrm{H}, \mathrm{d}, J$ $=7.8 \mathrm{~Hz}) ;{ }^{13} \mathrm{C} \mathrm{NMR}\left(75 \mathrm{MHz}, \mathrm{CDCl}_{3}\right): \delta 22.28,24.69,36.32,86.96,120.92,125.46$, 125.77, 128.90, 133.86, 154.88, 170.12; IR (KBr,_cm $\left.{ }^{-1}\right): 3042$ w, 2936 s, 2861 m, 1760 s, 1614 m, 1599 m, 1494 m, 1464 m, 1449 m, 1368 m, 1353 m, 1328 m, 1282 s, 1270 s, 1239 m, 1203 m, 1089 s, 1017 m, 946 s, 925 s, 861 m, 191 w, 760 s, 727 m, 693 s, 662 w, $601 \mathrm{~m}, 542 \mathrm{~m}$; MS, m/z: $202\left(\mathrm{M}^{+}\right)$.

From 3-phenethyl benzoic acid, two isomers, pthalide 4d (minor product) and isocoumarin 4d' (major product), were formed in total $81 \%$ yield (Eq. 1). These isomers were separable by usual column chromatography techniques $\left(\mathrm{SiO}_{2}\right.$ (neutral) $/ n$-hexane-AcOEt). The isolated yield of $\mathbf{4 d}$ and $\mathbf{4 d}$ ' is $18 \%$ and $63 \%$, respectively. Thus, the ratio of $\mathbf{4 d} / \mathbf{4} \mathbf{d}^{\prime}$ ' is calculated to 22:78. 


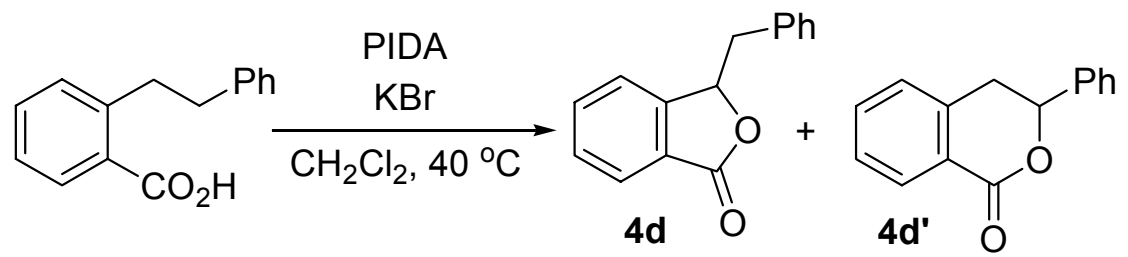

3-Phenylmethyl-1(3H)-isobenzofuranone (4d, minor product): ${ }^{17}$ white powder; m.p. $60-61{ }^{\circ} \mathrm{C}, R_{f}=0.36($ hexane/EtOAc $=3 / 1) ;{ }^{1} \mathrm{H}$ NMR $\left(270 \mathrm{MHz}, \mathrm{CDCl}_{3}\right): \delta 3.16(1 \mathrm{H}, \mathrm{dd}$, $J=14.1,6.2 \mathrm{~Hz}), 3.29(1 \mathrm{H}, \mathrm{dd}, J=14.1,6.8 \mathrm{~Hz}), 5.69(1 \mathrm{H}, \mathrm{t}, J=6.5 \mathrm{~Hz}), 7.12-7.81$ $(8 \mathrm{H}, \mathrm{m}), 7.84(1 \mathrm{H}, \mathrm{d}, J=7.6 \mathrm{~Hz}) ;{ }^{13} \mathrm{C}$ NMR $\left(75 \mathrm{MHz}, \mathrm{CDCl}_{3}\right): \delta 40.83,81.19,122.27$, $125.68,126.21,127.14,128.53,129.19,129.69,133.69,134.96,149.08,170.25$; IR $\left(\mathrm{KBr}, \mathrm{cm}^{-1}\right): 3030$ w, $2922 \mathrm{w}, 1765 \mathrm{~s}, 1601 \mathrm{w}, 1495 \mathrm{~m}, 1466 \mathrm{~m}, 1454 \mathrm{~m}, 1350 \mathrm{~m}, 1286$ m, 1267 m, 1213 m, 1155 w, 1065 s, 1015 m, 989 m, 912 w, 756 m, 737 m, 696 s, 627 w, $546 \mathrm{~m} ; \mathrm{MS}, \mathrm{m} / z: 224\left(\mathrm{M}^{+}\right)$.

3,4-Dihydro-3-phenyl-1H-2-benzopyran-1-one (4d', major product): ${ }^{18}$ white powder; m.p. 90-91 ${ }^{\circ} \mathrm{C}, R_{f}=0.41$ (hexane/EtOAc $\left.=3 / 1\right) ;{ }^{1} \mathrm{H}$ NMR $\left(300 \mathrm{MHz}, \mathrm{CDCl}_{3}\right)$ : $\delta 3.14(1 \mathrm{H}, \mathrm{dd}, J=16.5,3.3 \mathrm{~Hz}), 3.36(1 \mathrm{H}, \mathrm{dd}, J=16.5,12.0 \mathrm{~Hz}), 5.57(1 \mathrm{H}, \mathrm{dd}, J=$ 12.0, $3.3 \mathrm{~Hz}), 7.00-7.60(3 \mathrm{H}, \mathrm{m}), 8.16(1 \mathrm{H}, \mathrm{d}, J=7.8 \mathrm{~Hz}) ;{ }^{13} \mathrm{C} \mathrm{NMR}(75 \mathrm{MHz}$, $\left.\mathrm{CDCl}_{3}\right): \delta 35.62,79.97,125.11,126.11,127.34,127.88,128.65,128.68,130.43$, 133.91, 138.52, 138.92, 165.34; IR (KBr, cm $\left.{ }^{-1}\right): 3034$ w, 2924 w, 2853 w, 1728 s, 1605 m, 1487 w, 1421 w, 1346 m, 1271 s, 1227 m, 1119 s, 1084 s, 1067 m, 1030 m, 1001 m, $912 \mathrm{~m}, 800 \mathrm{w}, 743 \mathrm{~s}, 698 \mathrm{~s}, 640 \mathrm{w} ; \mathrm{MS}, \mathrm{m} / z: 224\left(\mathrm{M}^{+}\right)$.

\section{References}

1) Kazmierczak, P.; Skulski, L.; Kraszkiewicz, L. Molecules 2001, 6, 881.

2) Miyano, S.; Tatsuoka, T.; Suzuki, K.; Imao, K.; Satoh, F.; Ishihara, T.; Hirotsu, I.; 
Kihara, T.; Hatta, M.; Horikawa, Y.; Sumoto, K. Chem. Pharm. Bull. 1990, 38, 1570.

3) Vebrel, J.; Carrie, R. Bull. Soc. Chim. Fr. 1982, 116.

4) Yus, M.; Torregrosa, R.; Pastor, I. M. Molecules 2004, 9, 330.

5) Bietti, M.; Capone, A. J. Org. Chem. 2006, 71, 5260.

6) Nakamura, E.; Oshino, H.; Kuwajima, I. J. Am. Chem. Soc. 1986, 108, 3745.

7) Verdaguer, X.; Hansen, M. C.; Berk, S. C.; Buchwald, S. L. J. Org. Chem. 1997, 62, 8522.

8) Sato, M.; Kosasayama, A.; Uchimaru, F. Chem. Pharm. Bull. 1981, 29, 2885.

9) Mahmoodi, N. O.; Jazayri, M. Synth. Commun. 2001, 31, 1467.

10) Lehmann, J.; Marquardt, N. Synthesis 1987, 1064.

11） Fang, J. M.; Hong, B. C.; Liao, L. F. J. Org. Chem. 1987, 52, 855.

12) Theil, F.; Costisella, B.; Gross, H.; Schick, H.; Schwarz, S. J. Chem. Soc., Perkin Trans. 1 1987, 2469.

13) Hirano, M.; Ueno, Y.; Morimoto, T. Synth. Commun. 1995, 25, 3765.

14） Knepper, K.; Ziegert, R. E.; Brase, S. Tetrahedron 2004, 60, 8591.

15) Fuji, K.; Morimoto, T.; Tsutsumi, K.; Kakiuchi, K. Chem. Commun. 2005, 3295.

16) Canonne, P.; Belanger, D.; Lemay, G.; Foscolos, G. B. J. Org. Chem. 1981, 46, 3091.

17） Canonne, P.; Plamondon, J.; Akssira, M. Tetrahedron 1988, 44, 2903.

18） Fu, P. P.; Unruh, L. E.; Miller, D. W.; Huang, L. W.; Yang, D. T. C. J. Org. Chem. 1985, 50, 1259. 

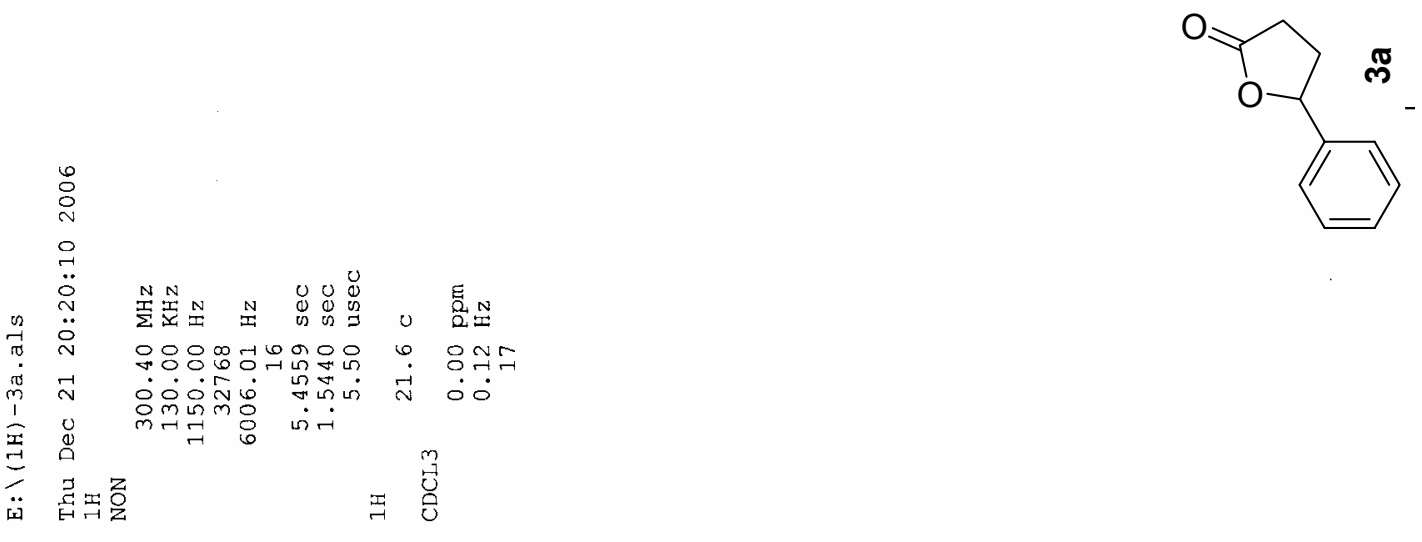

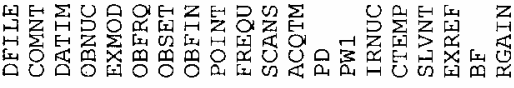

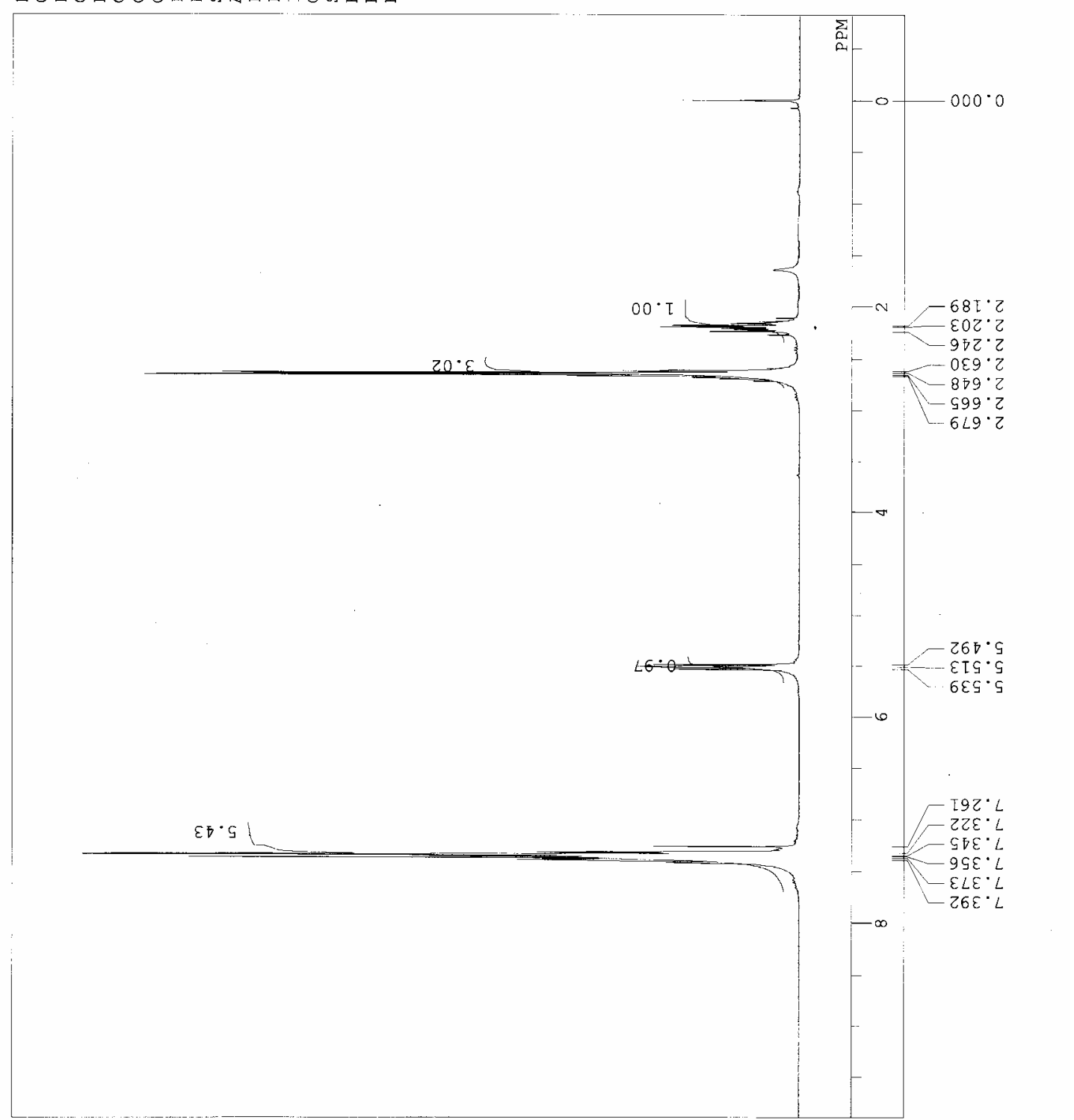



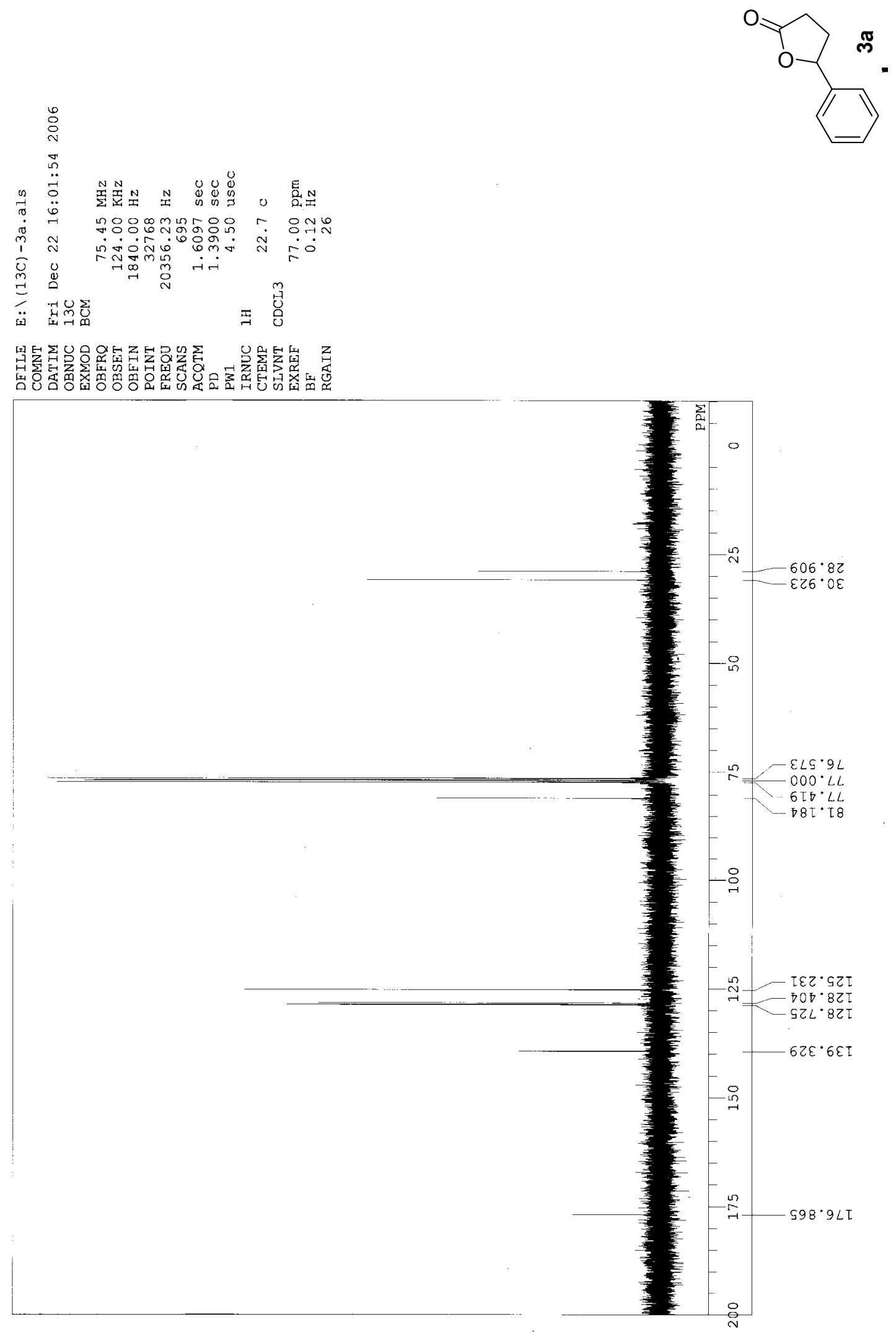

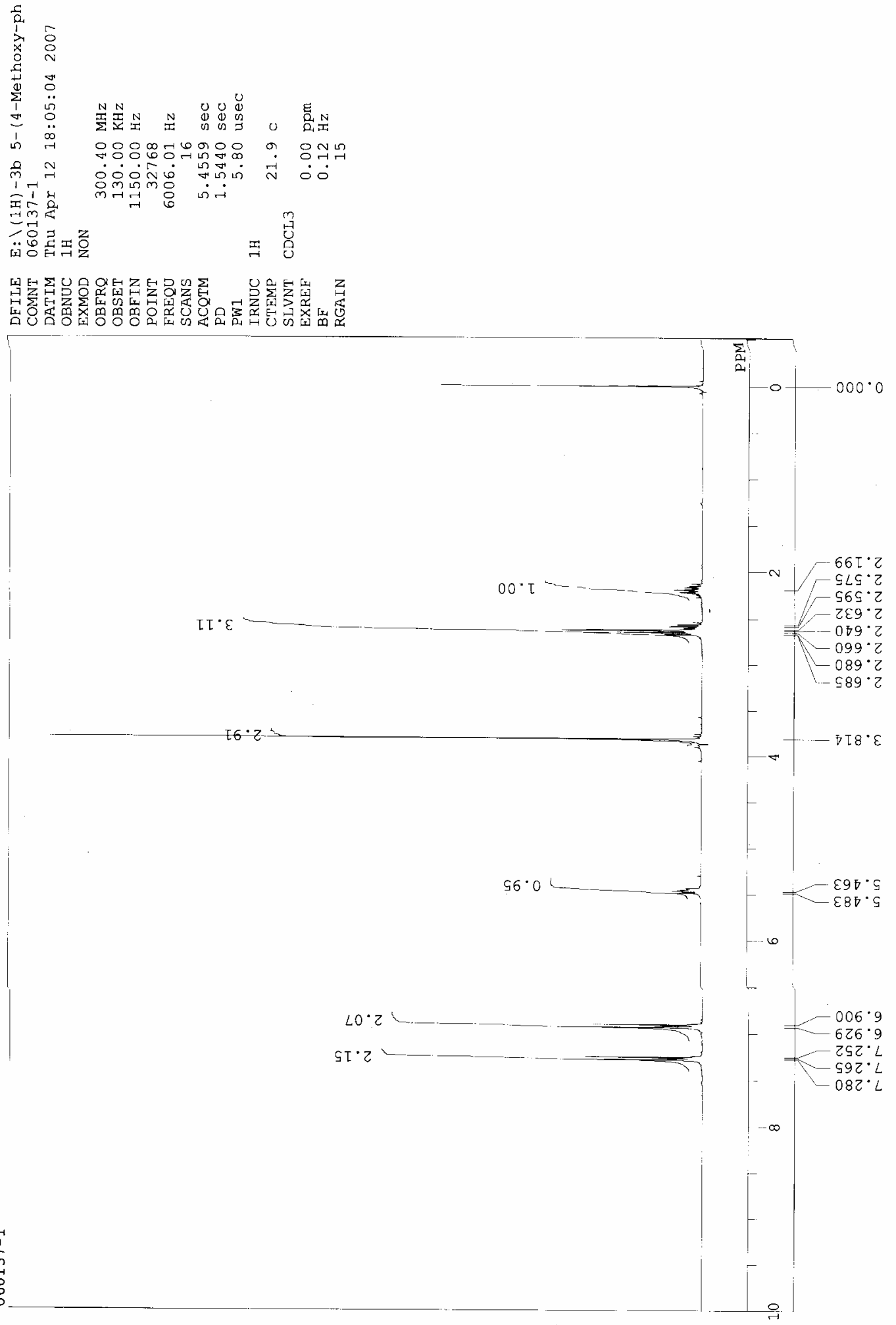


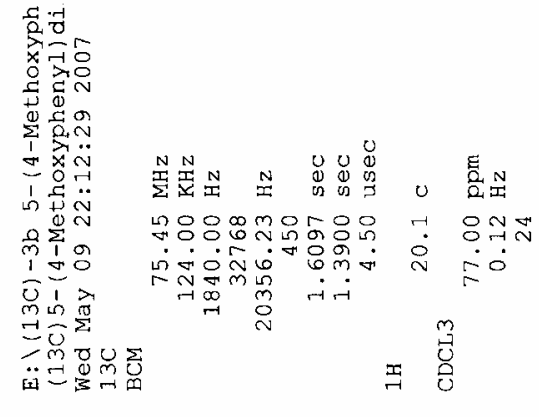

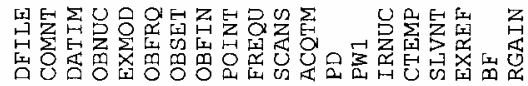

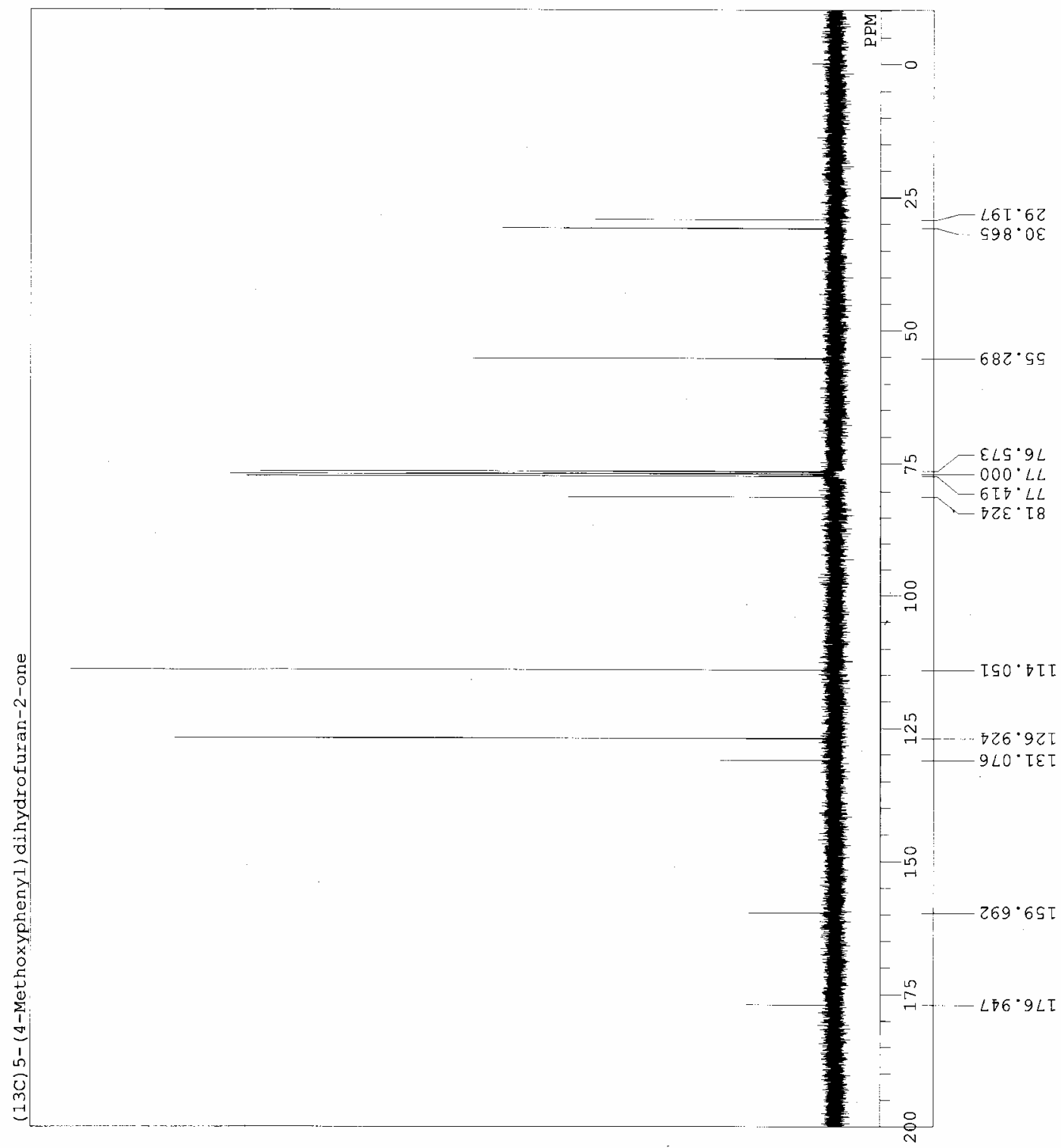



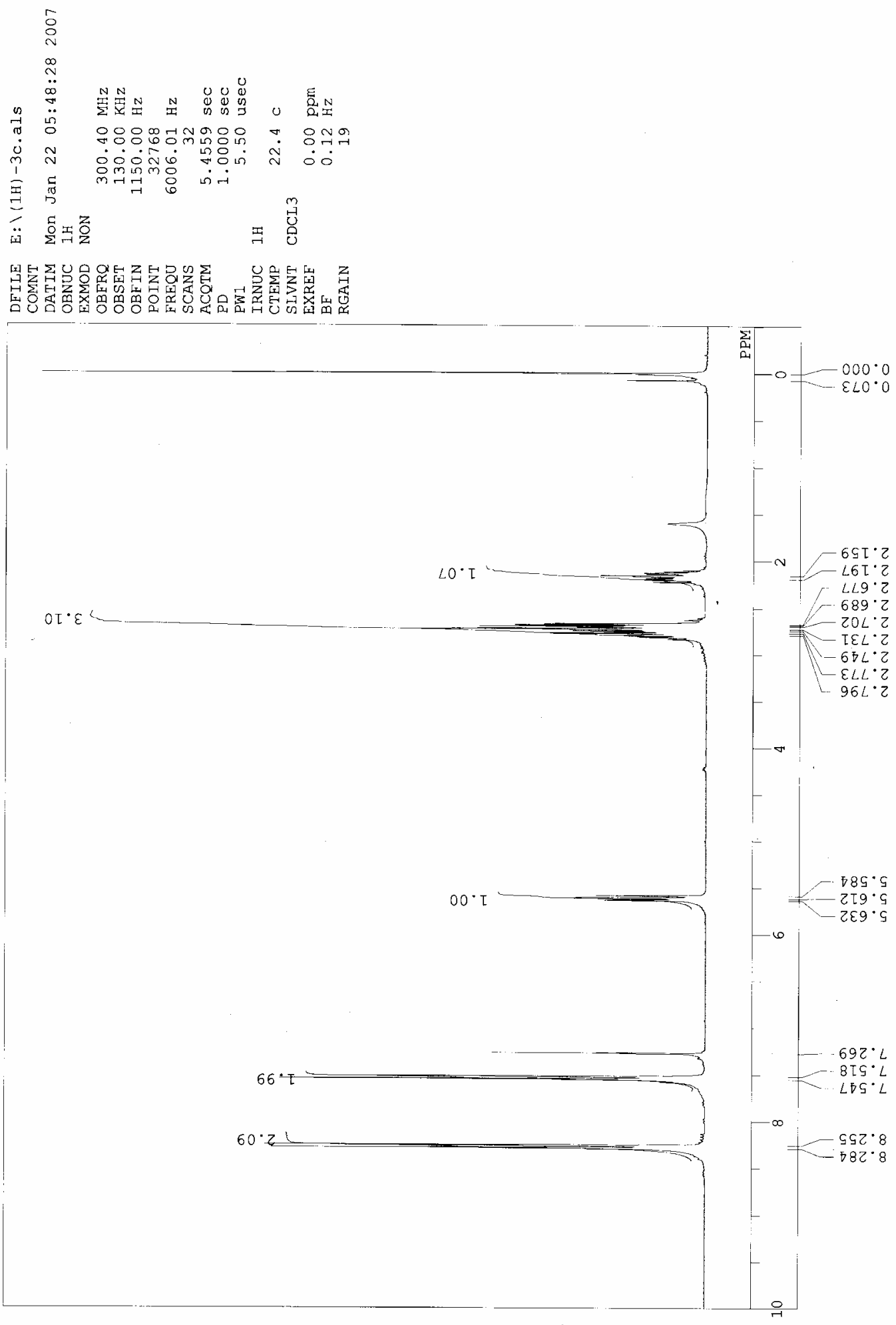


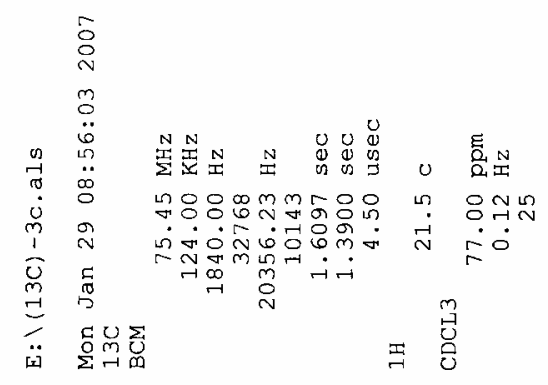

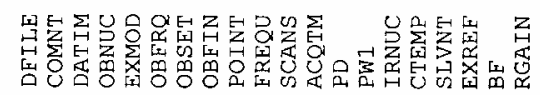

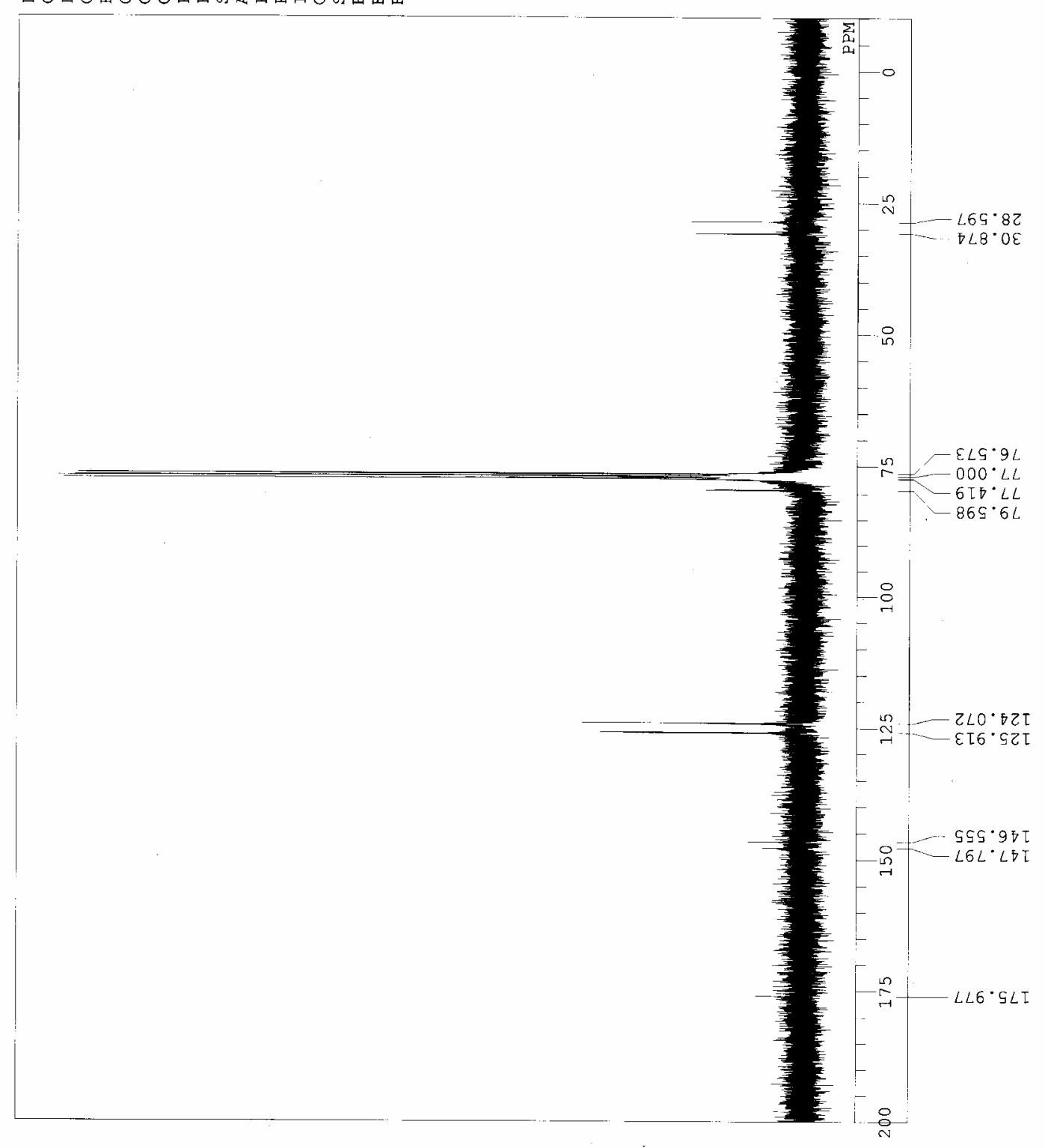




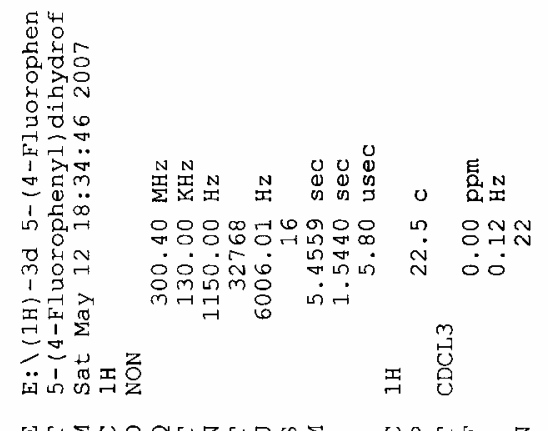

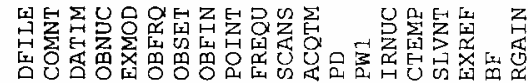

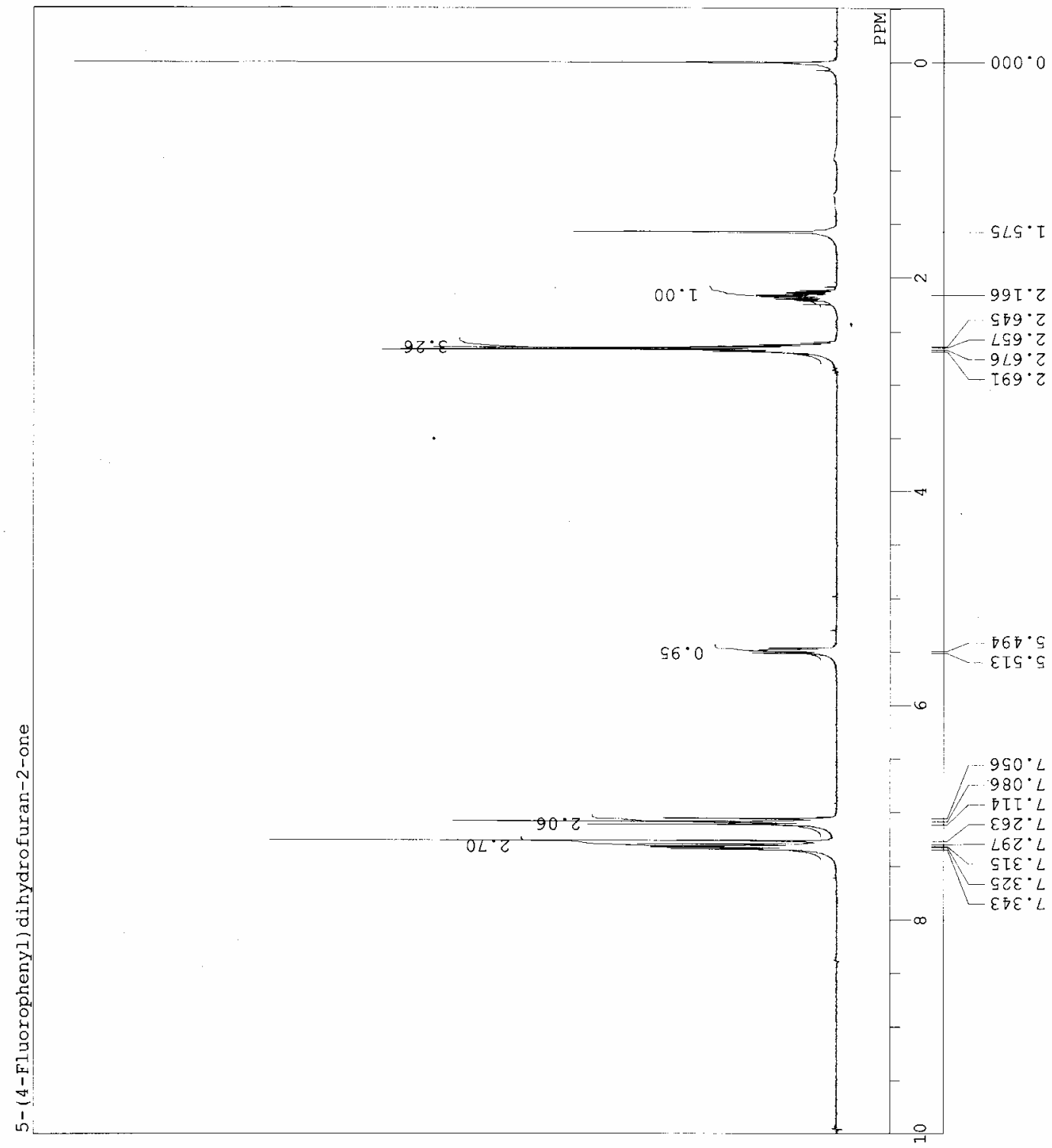




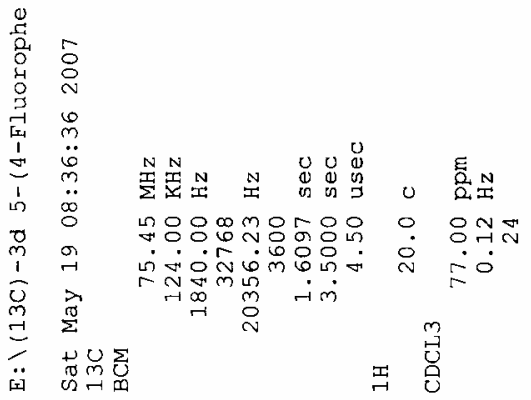

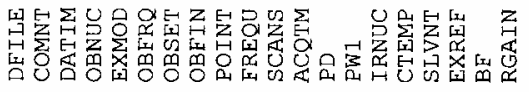

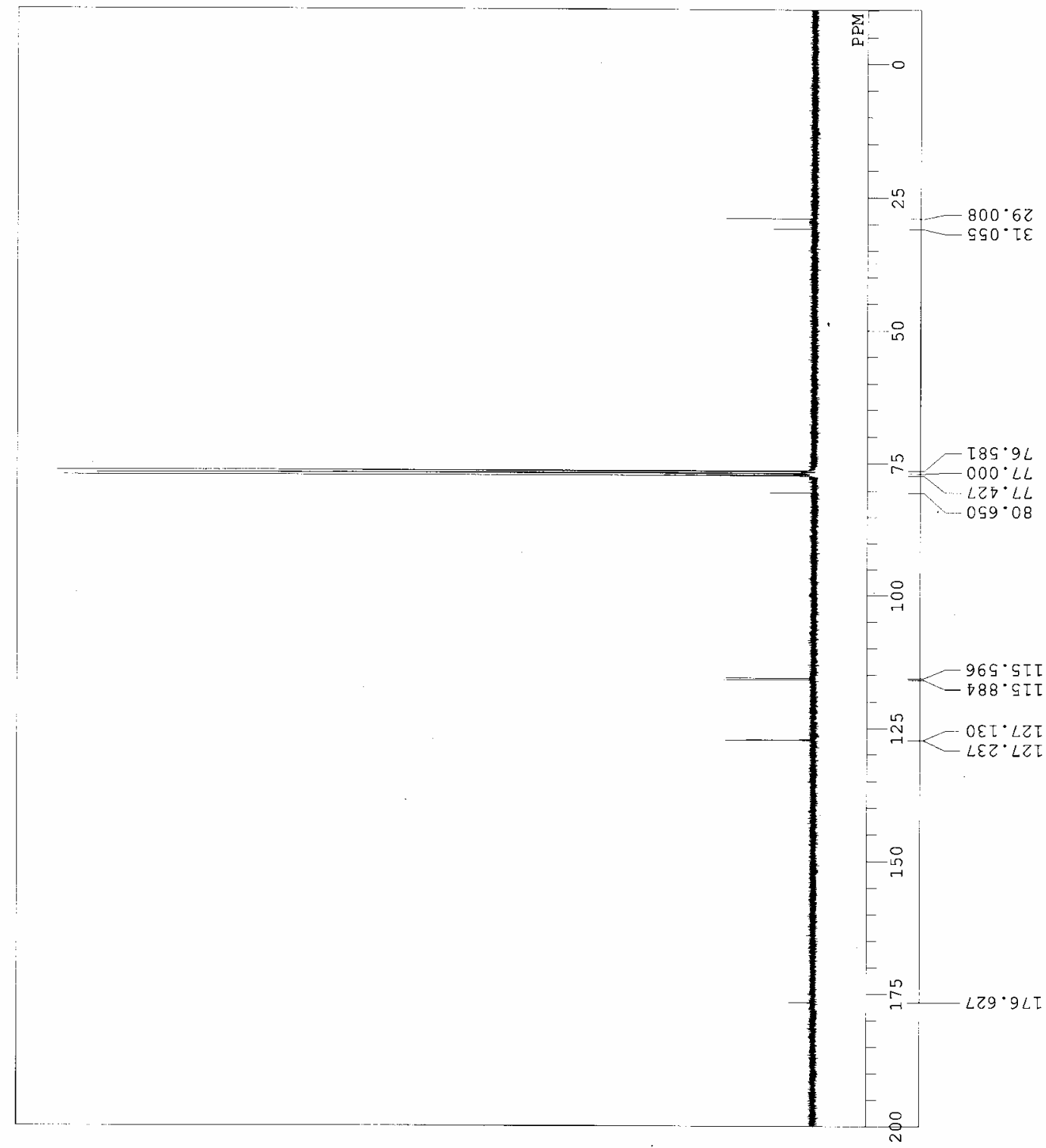




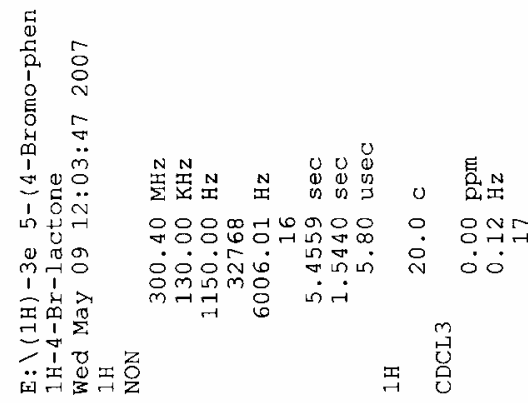

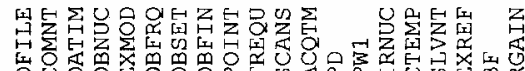

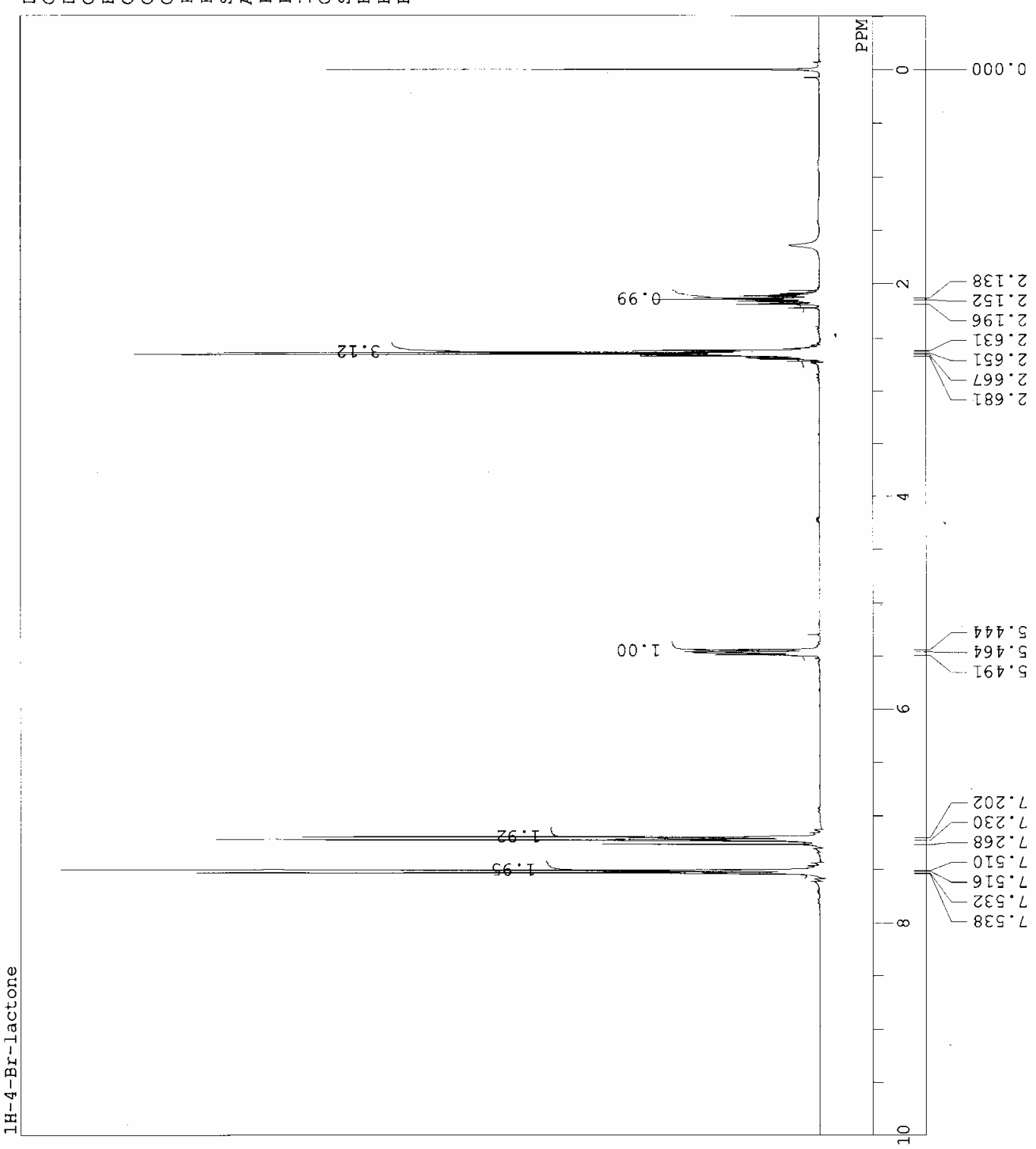


$\begin{array}{cc}0 & \\ 0 & \\ 0 & \\ 1 & 0 \\ 0 & 0 \\ 0 & 0 \\ 0 & 0 \\ 0 & 0 \\ 1 & 0 .\end{array}$

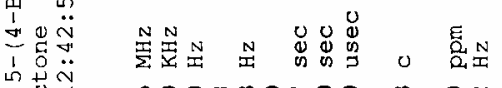

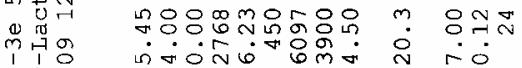

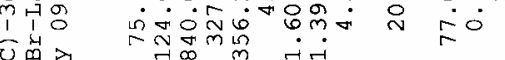

प्रm

$\exists$

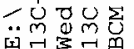

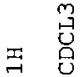

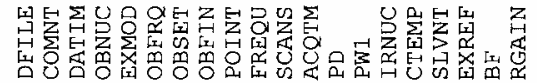

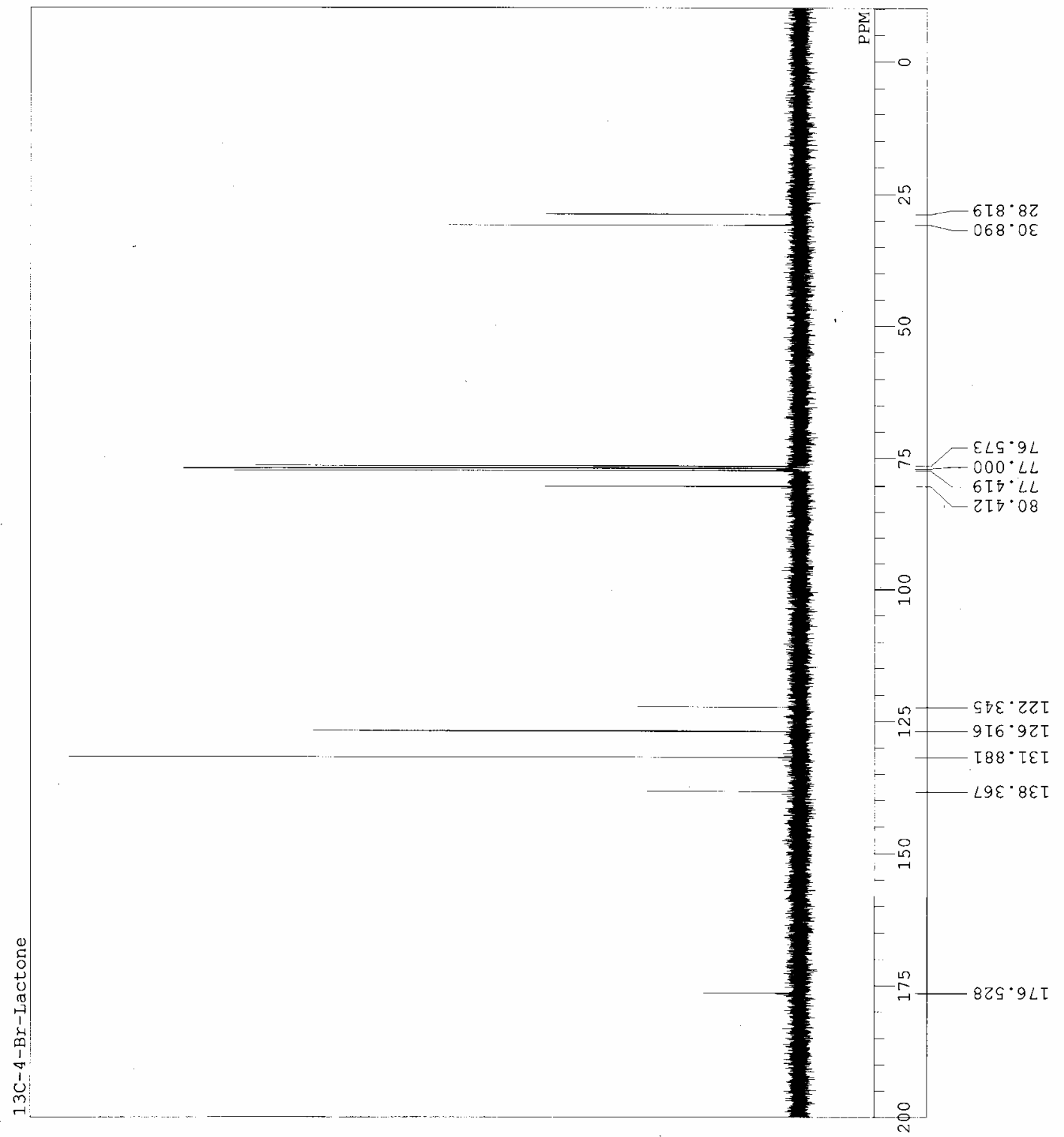



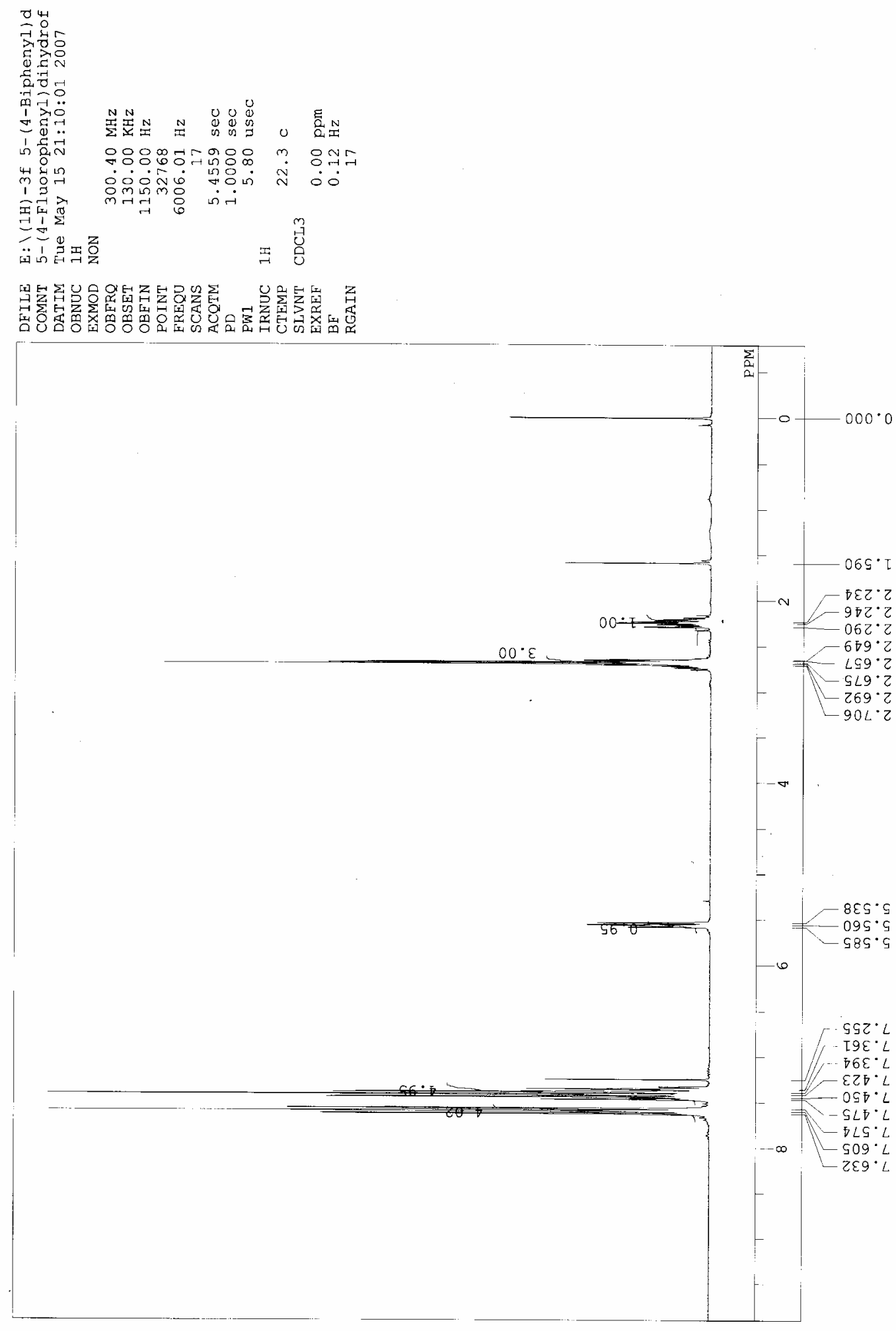


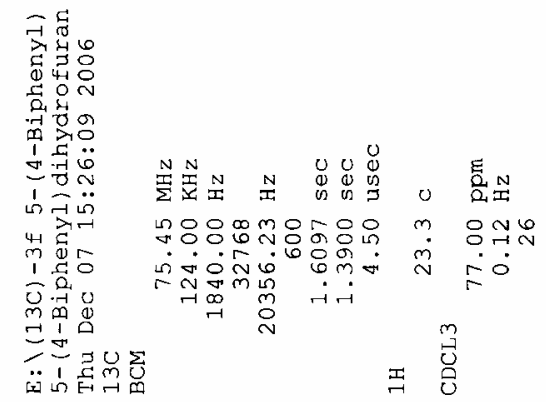

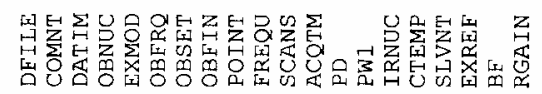

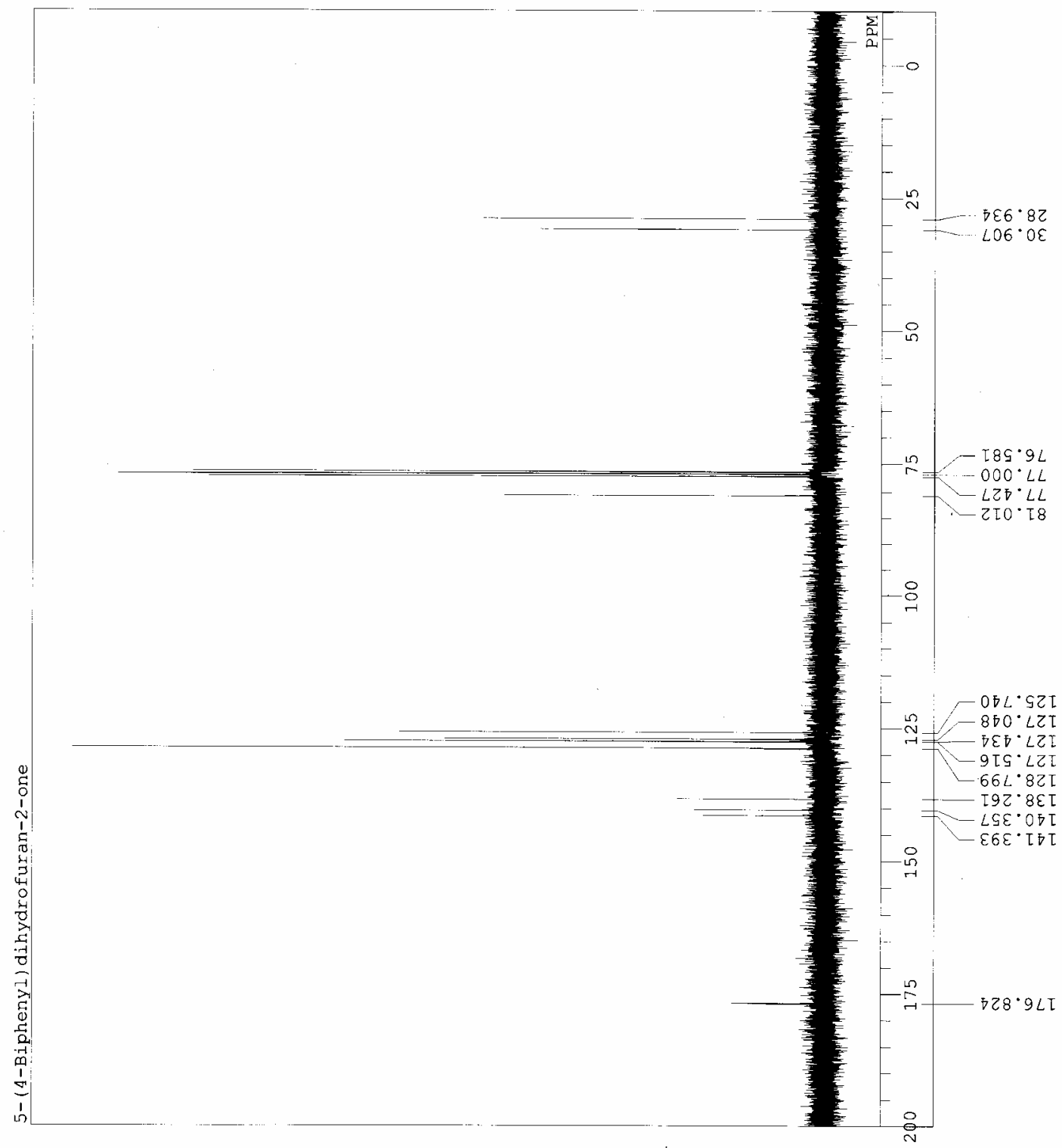




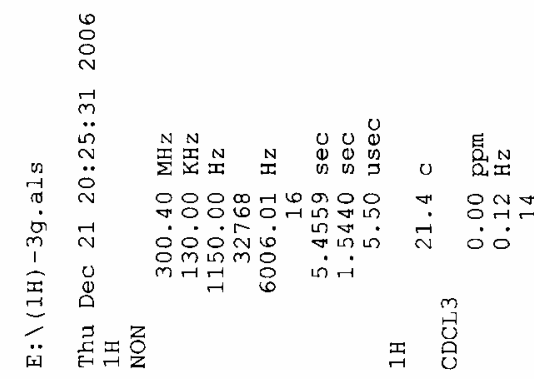

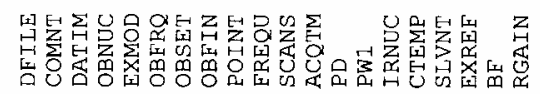

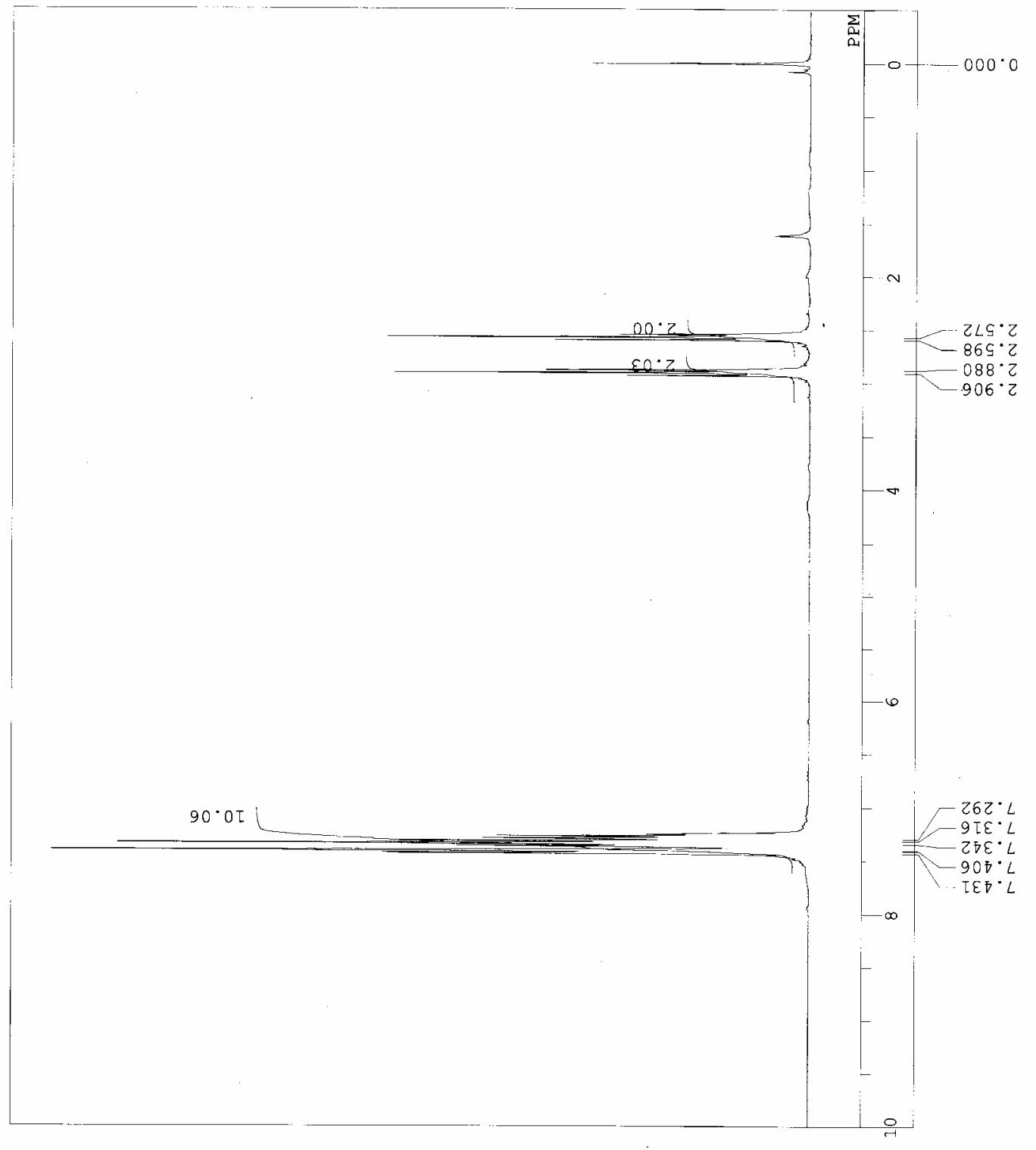




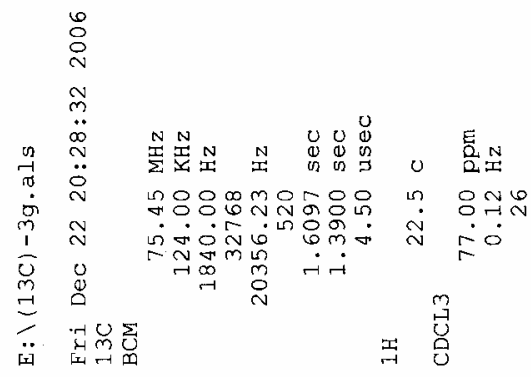

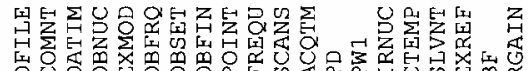

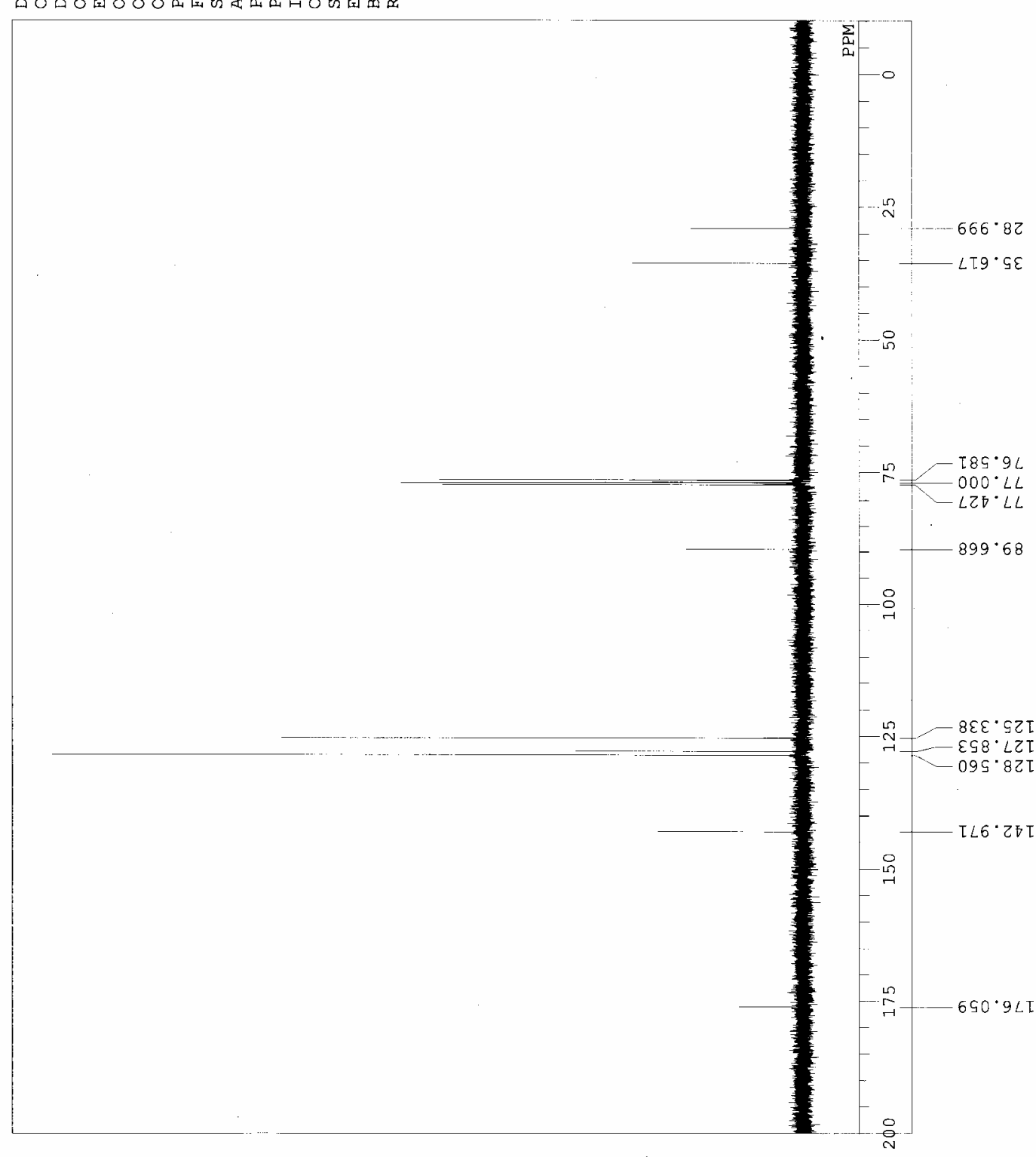




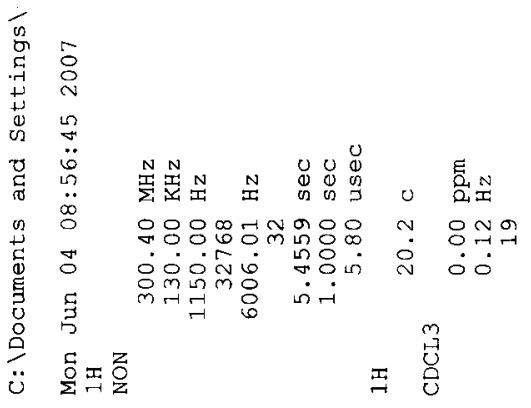

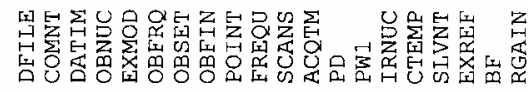

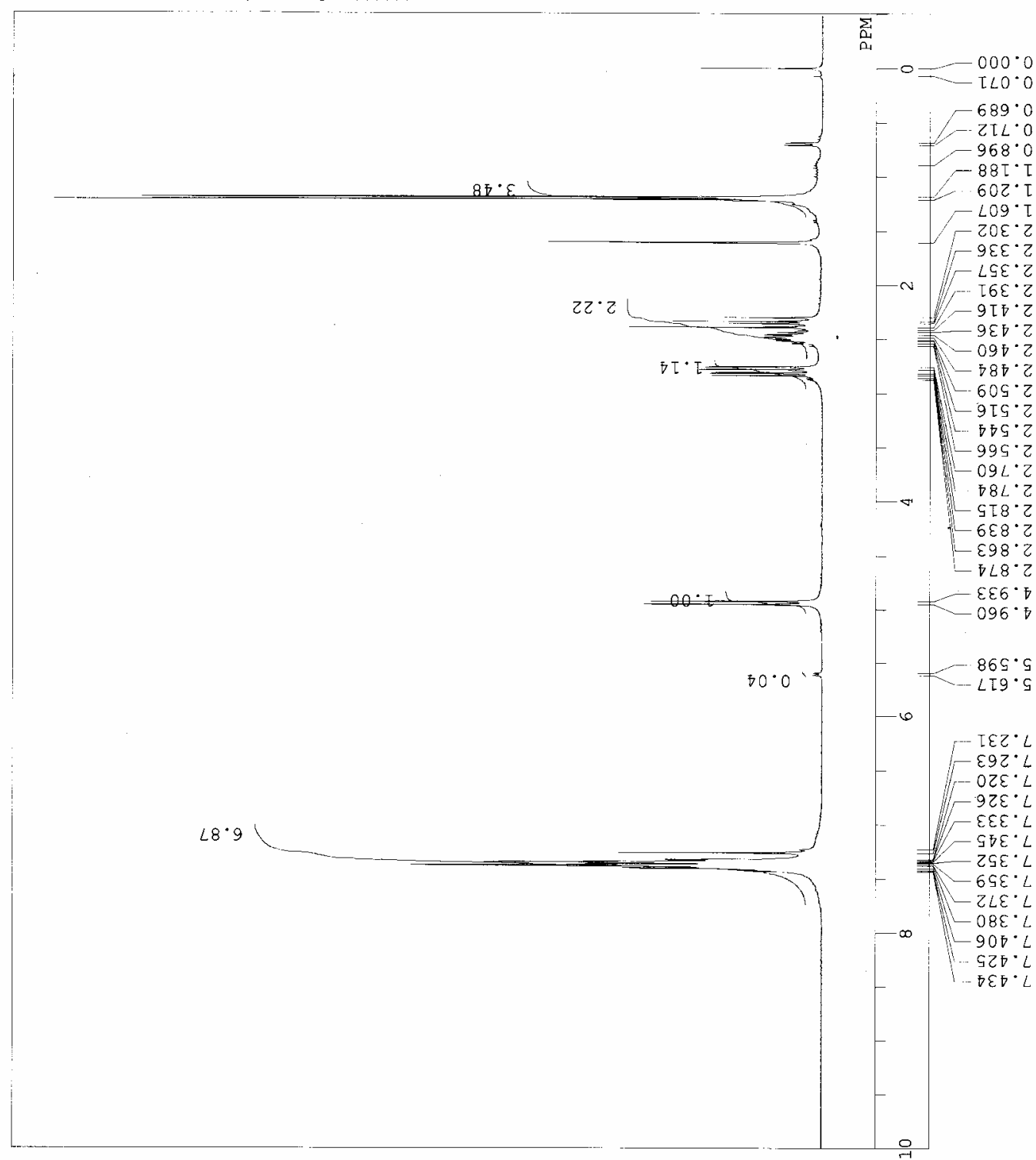




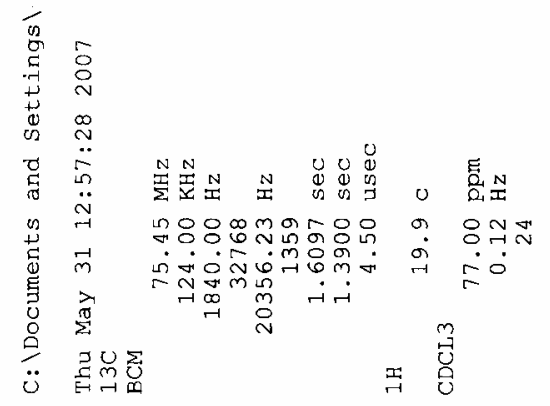

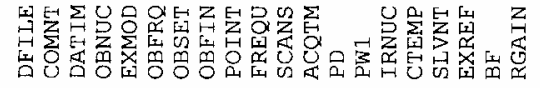

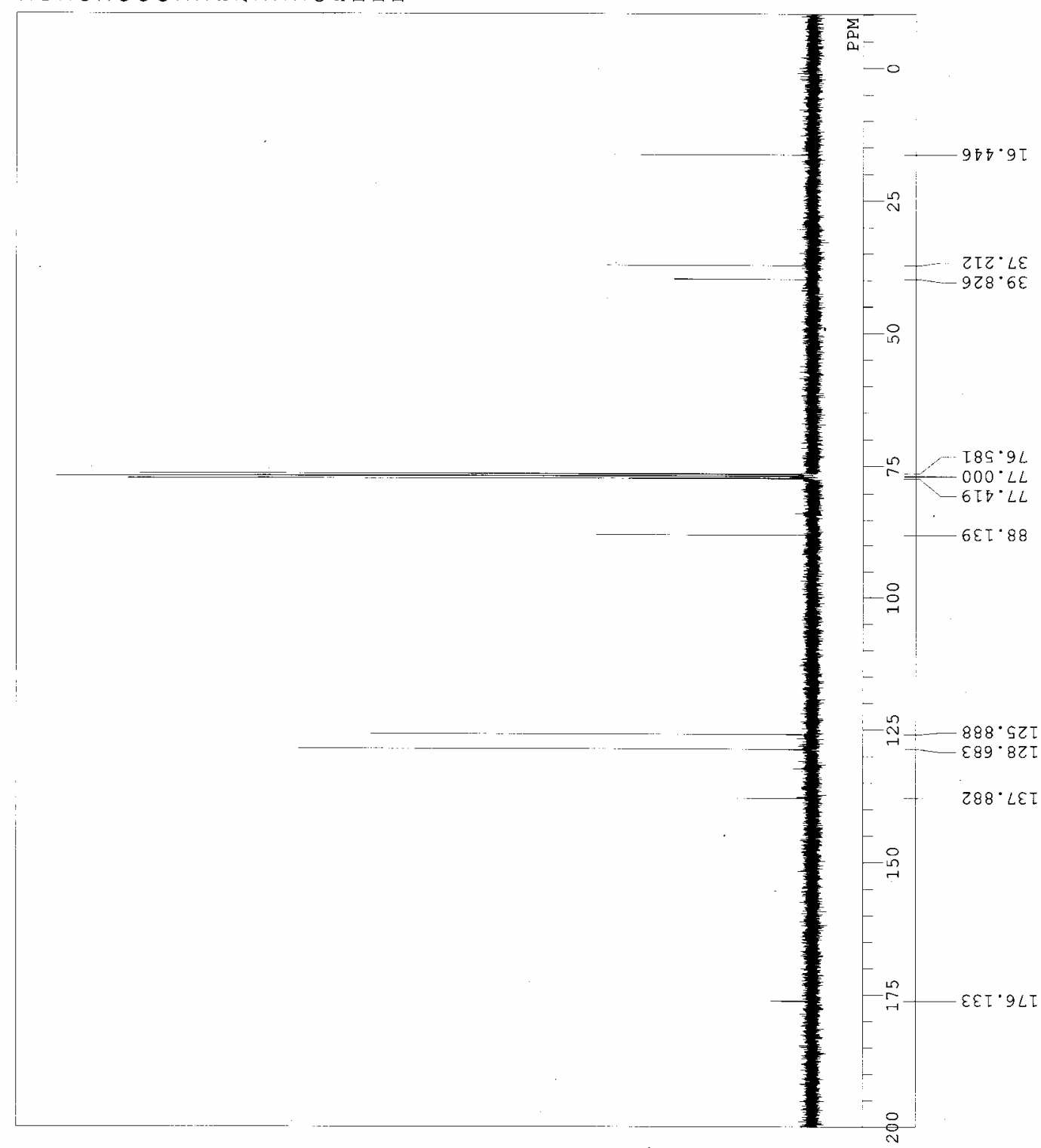




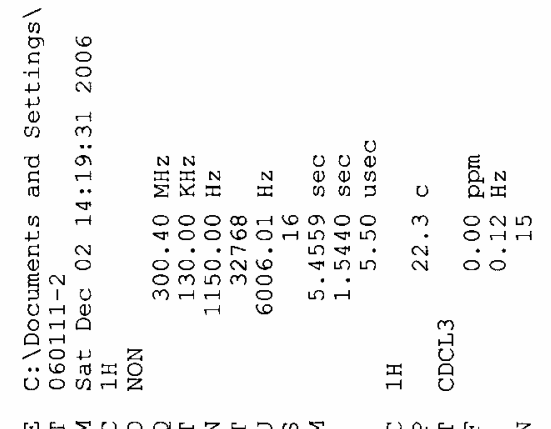

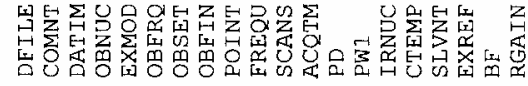

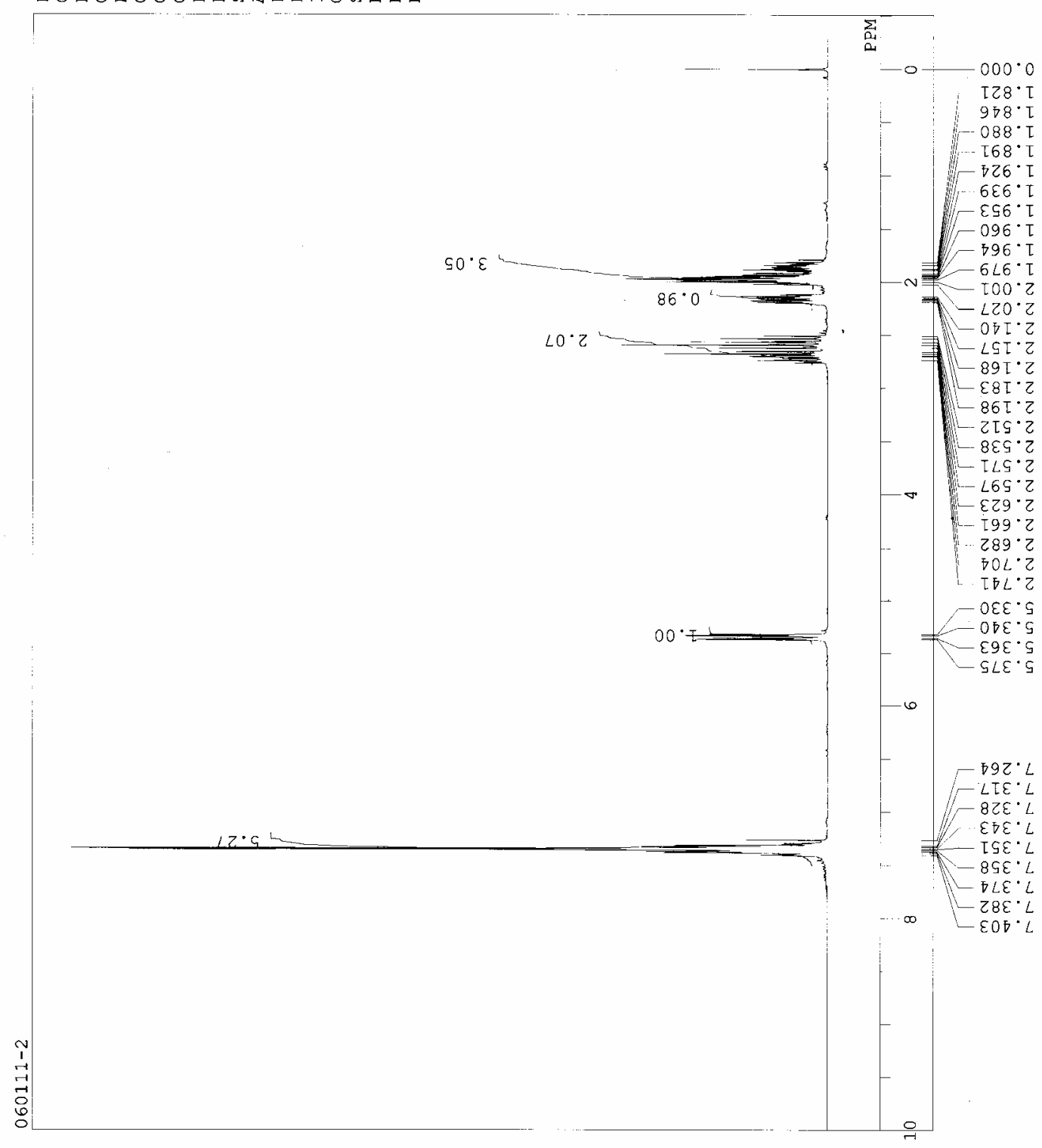




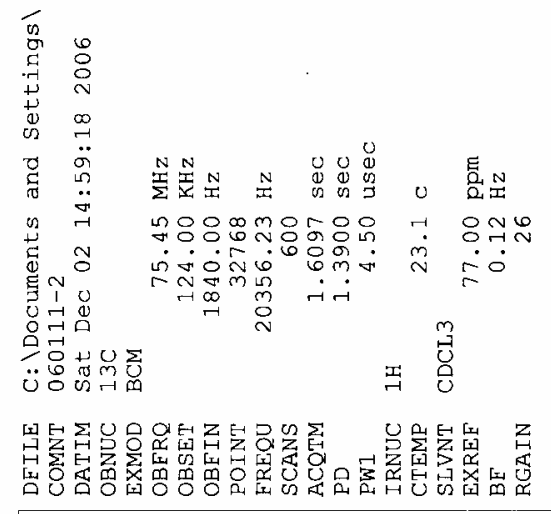

1
1
7
7
7
8
0

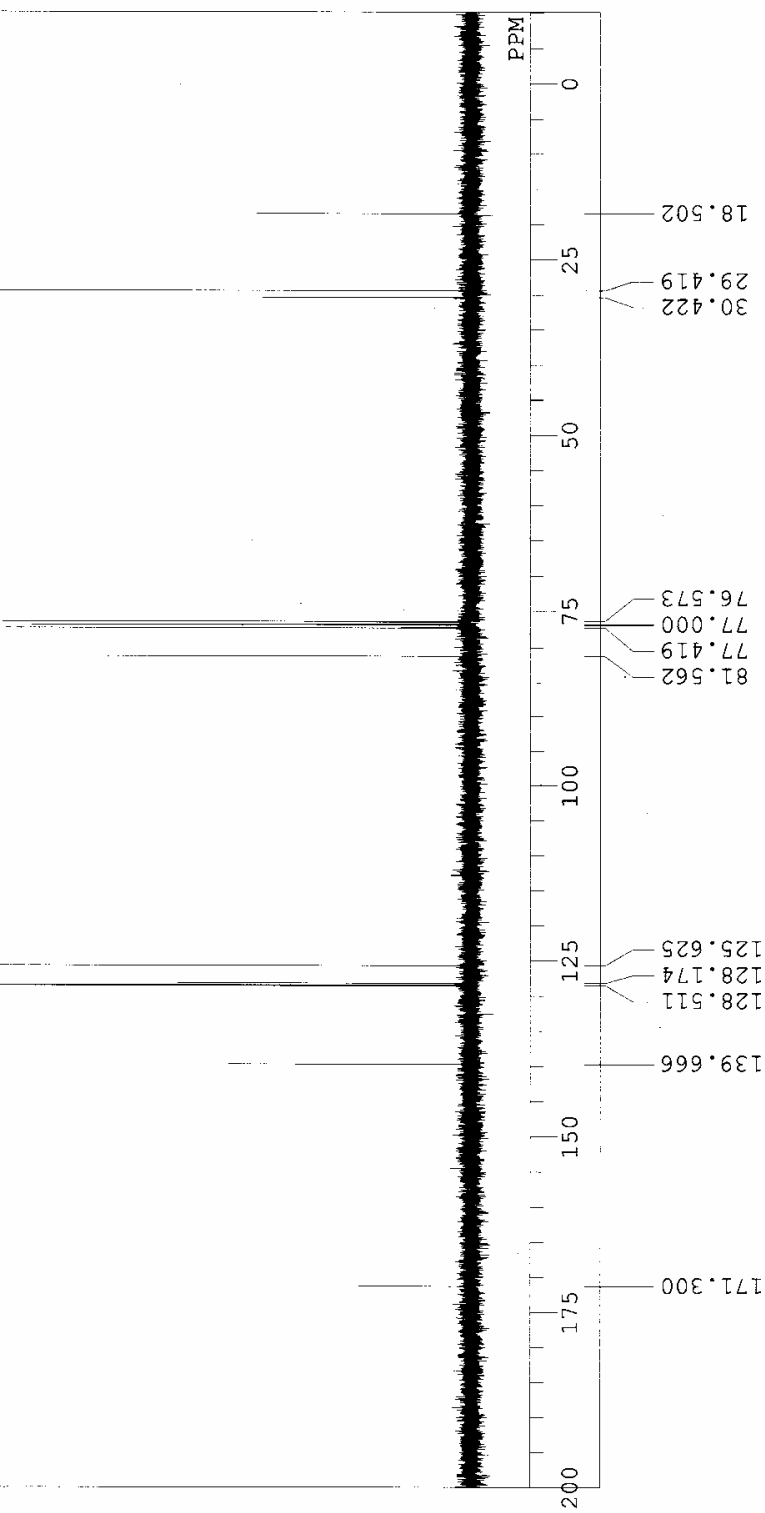




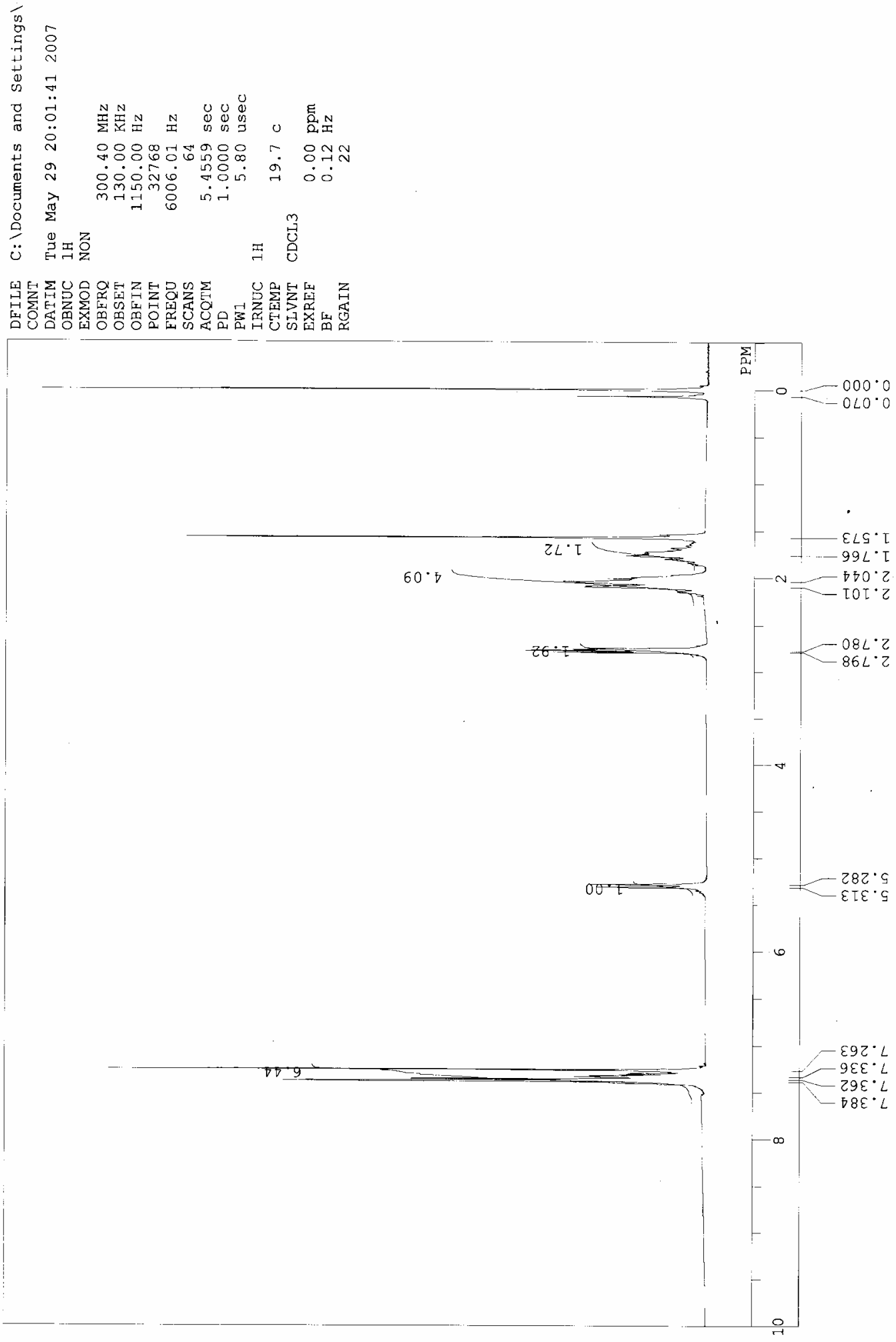




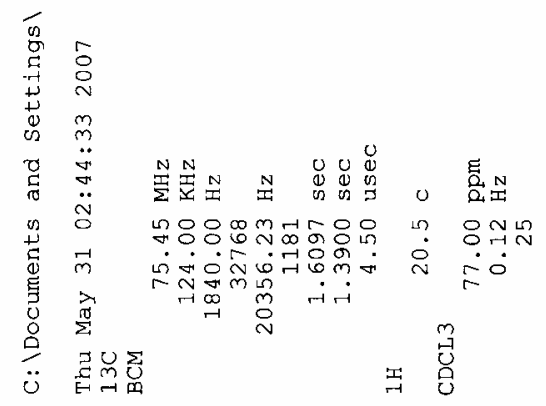

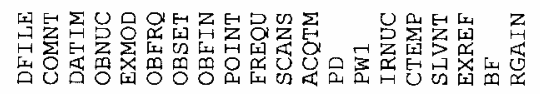

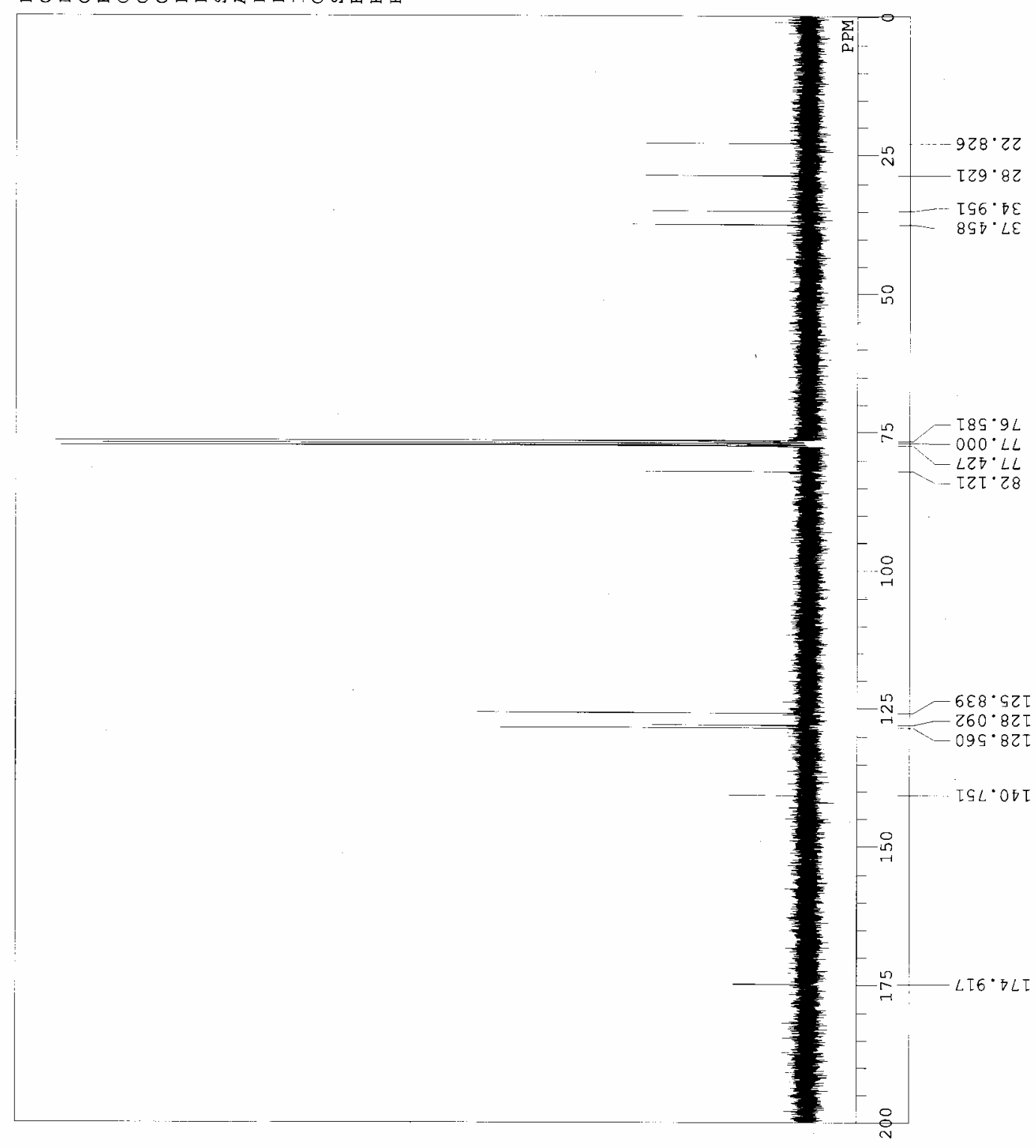




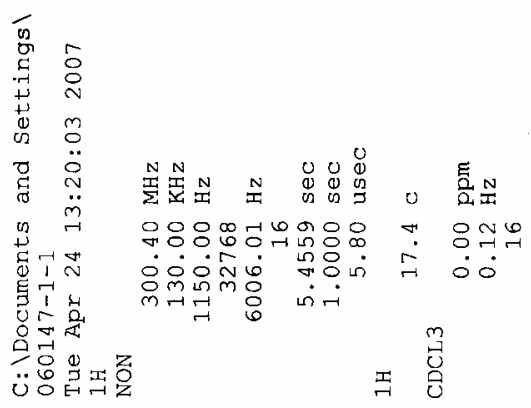

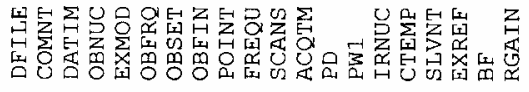

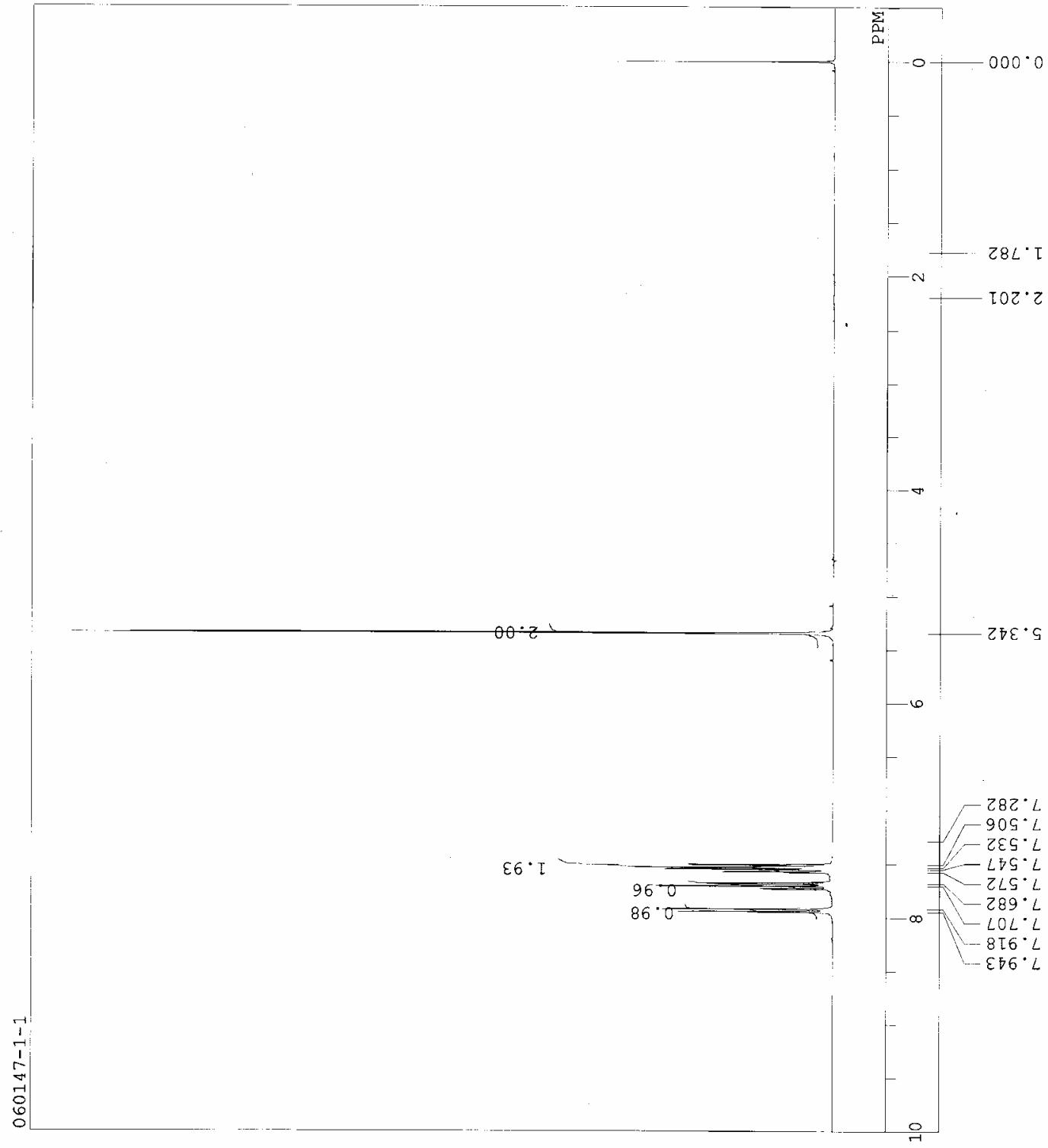




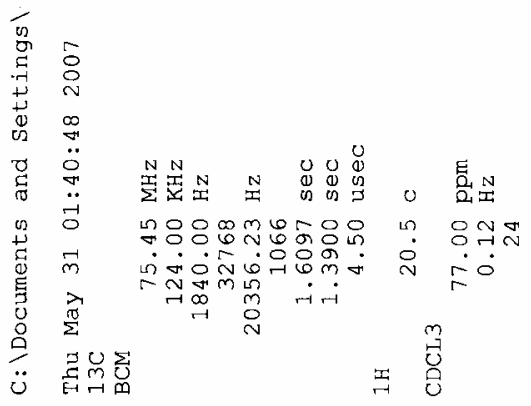

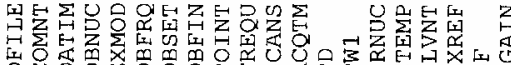

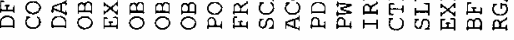

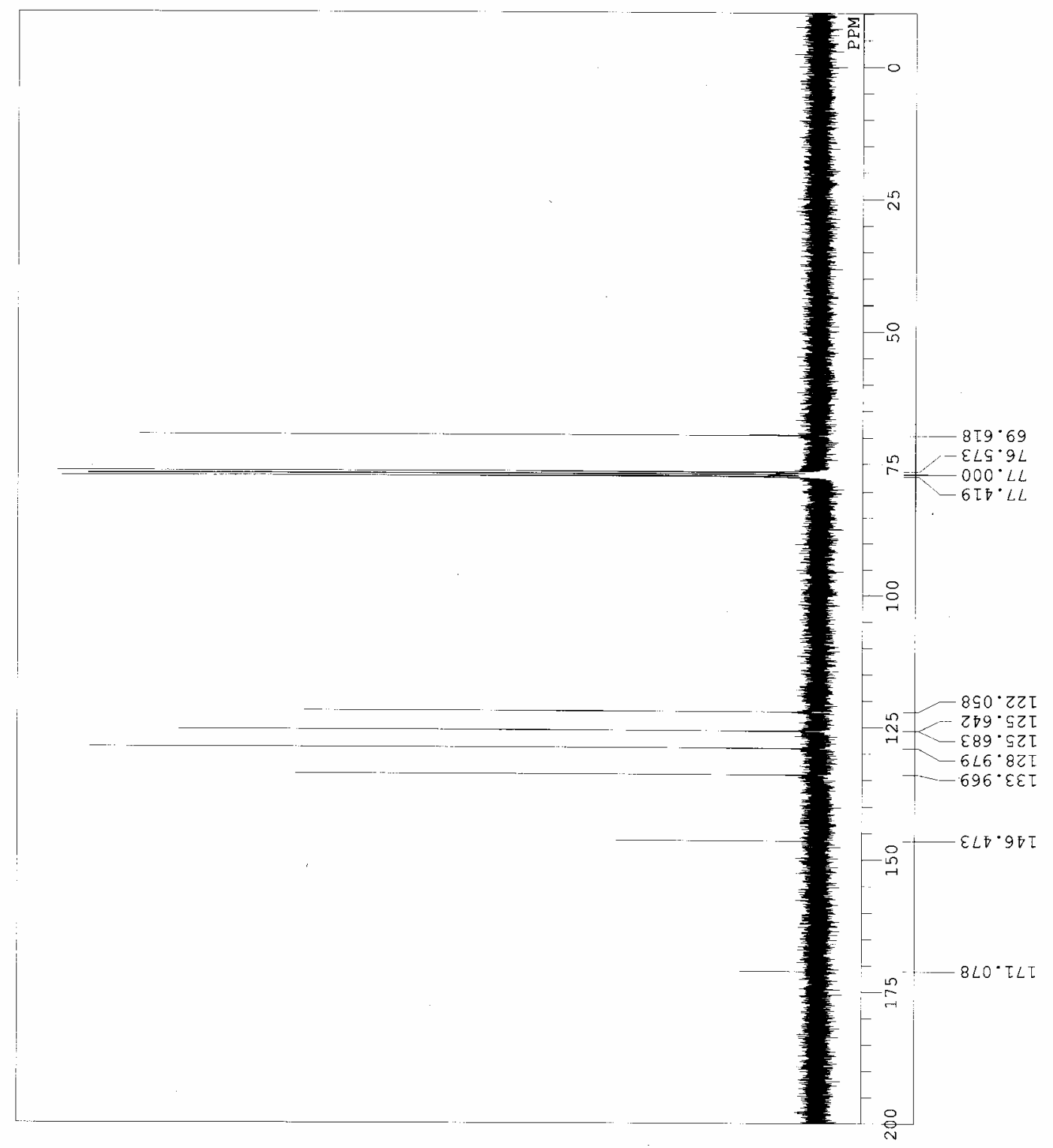




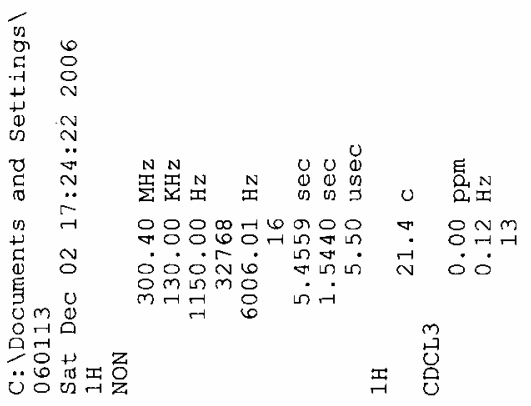

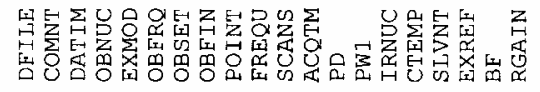

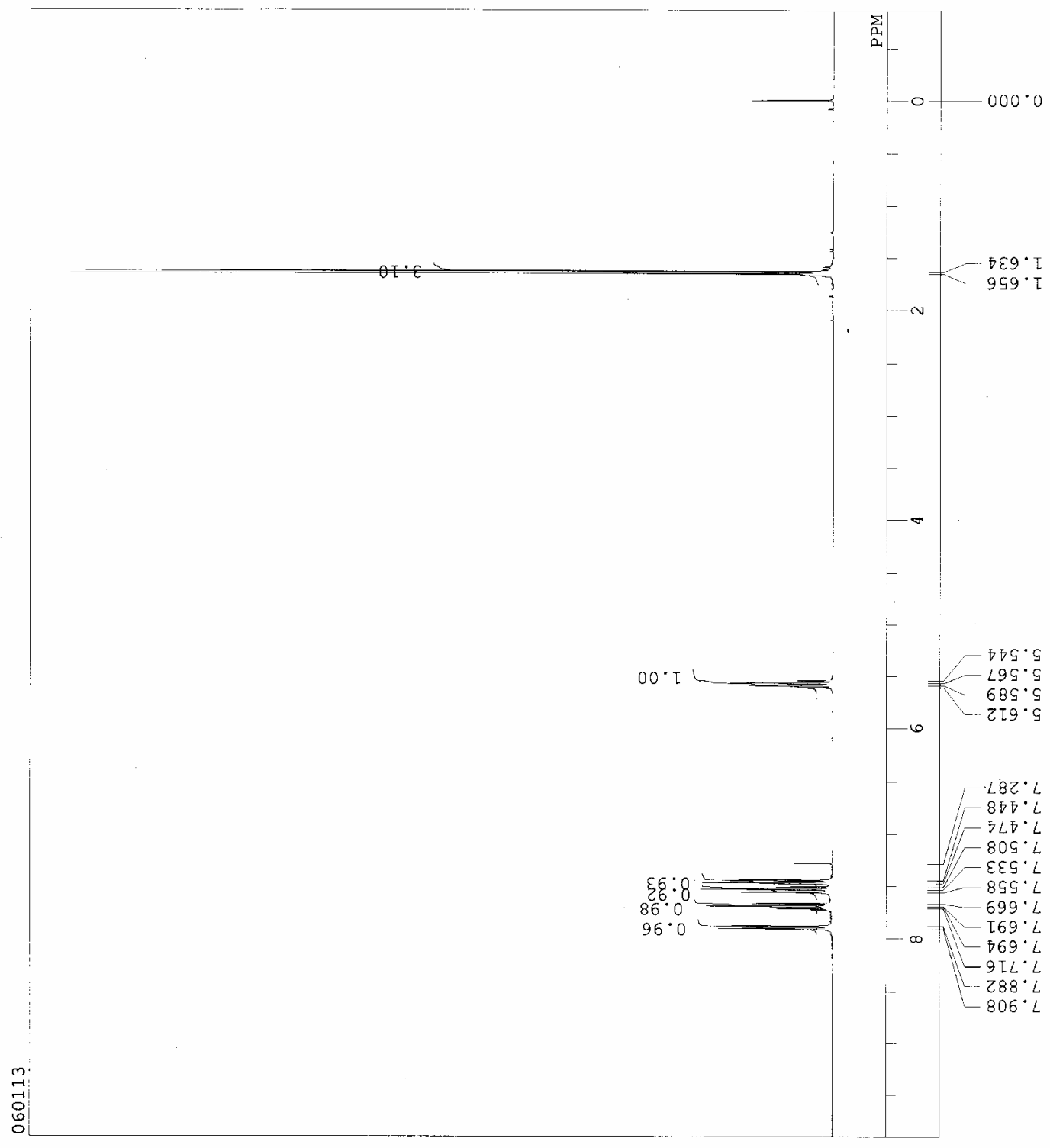




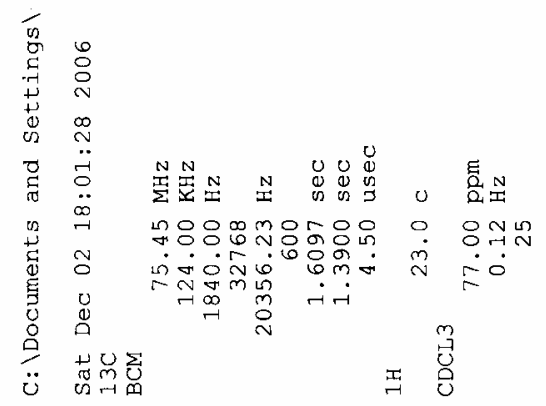

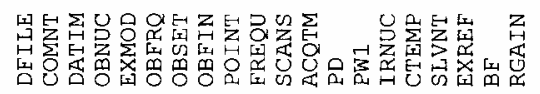

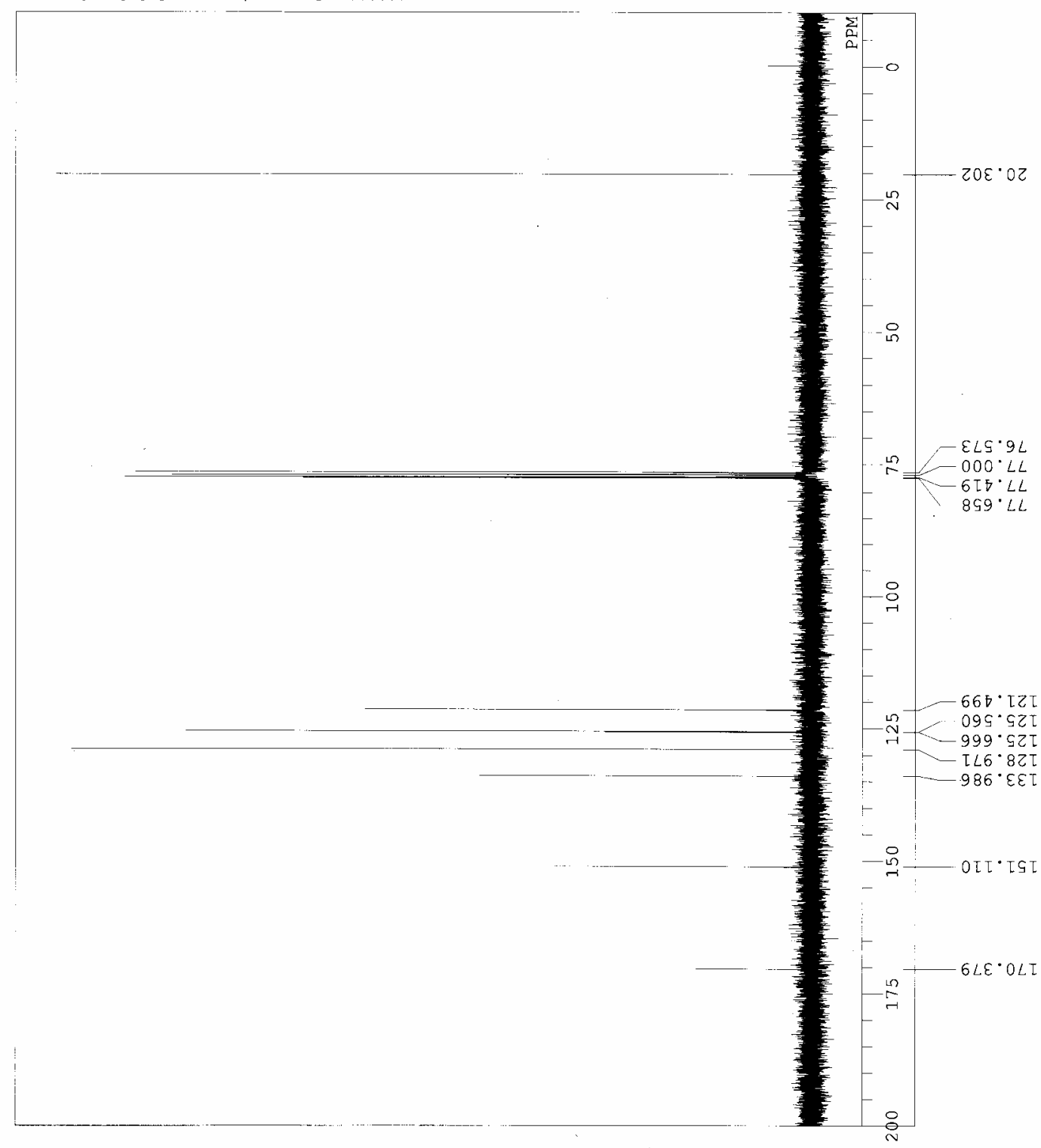



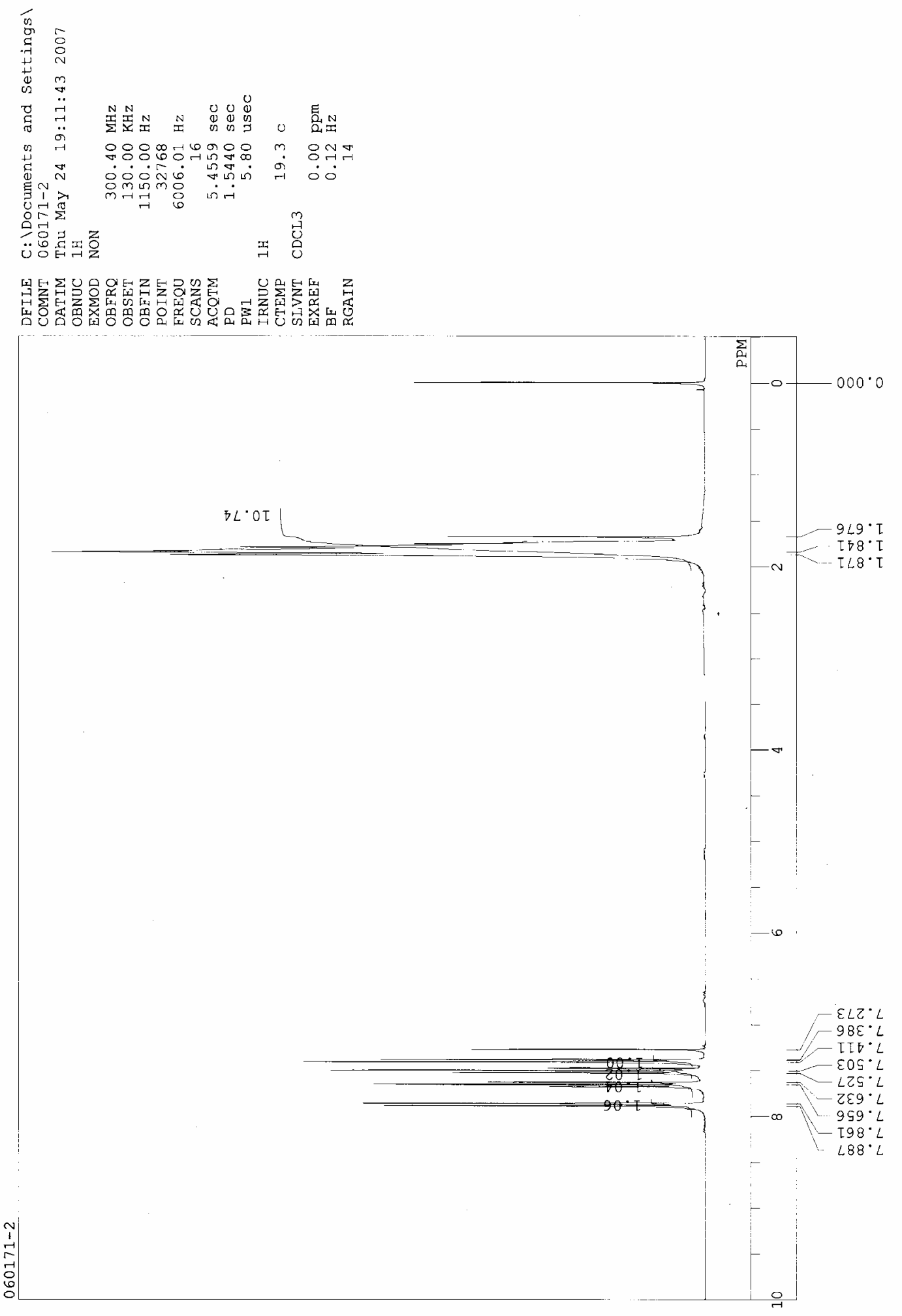


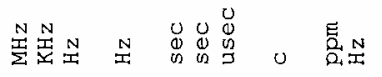

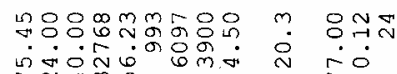

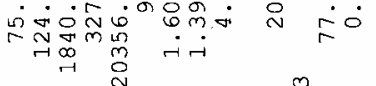

高

픔

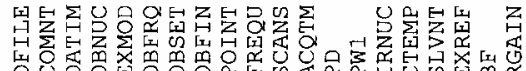

作

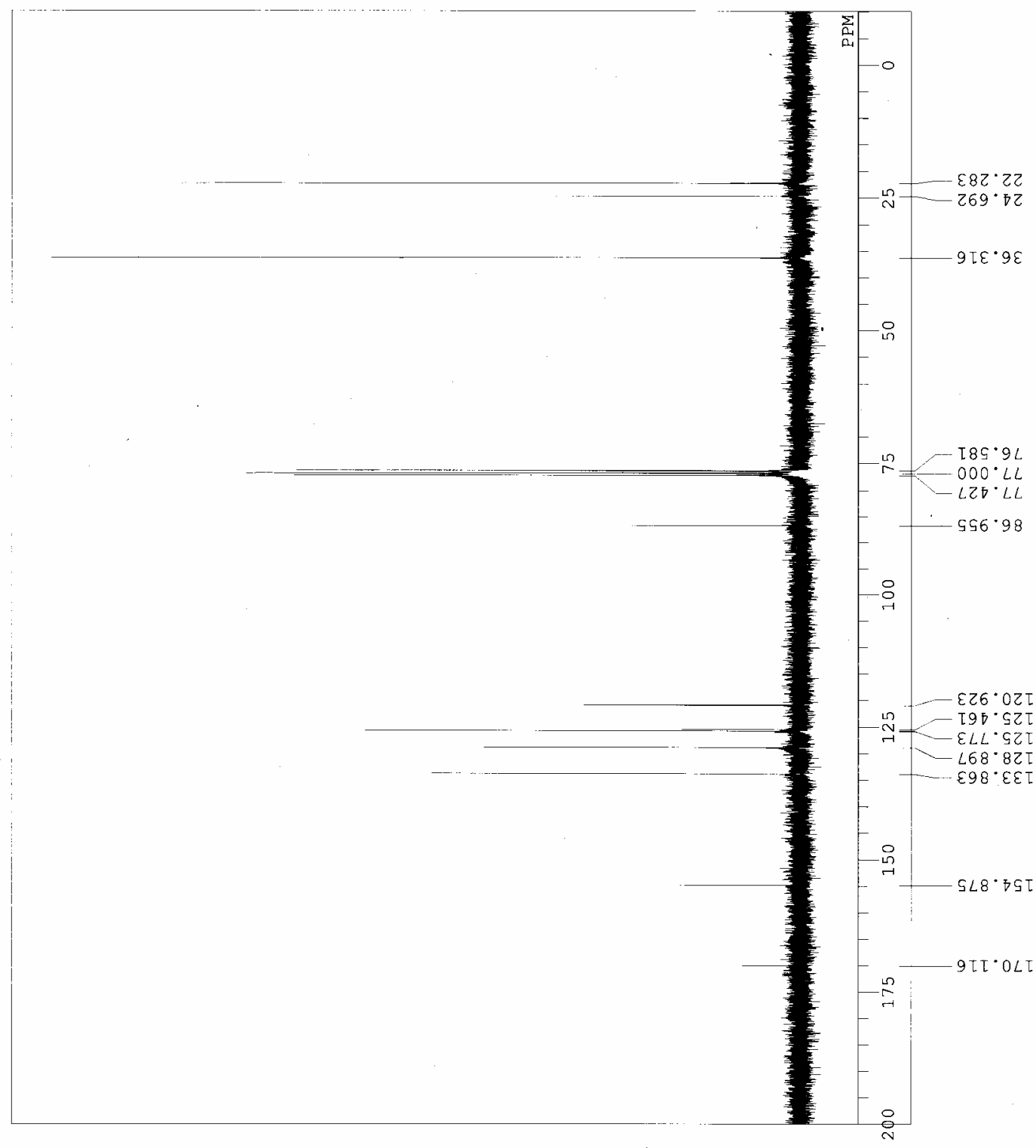




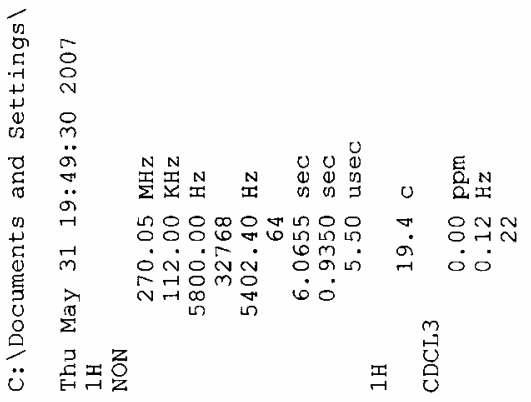

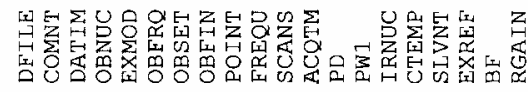

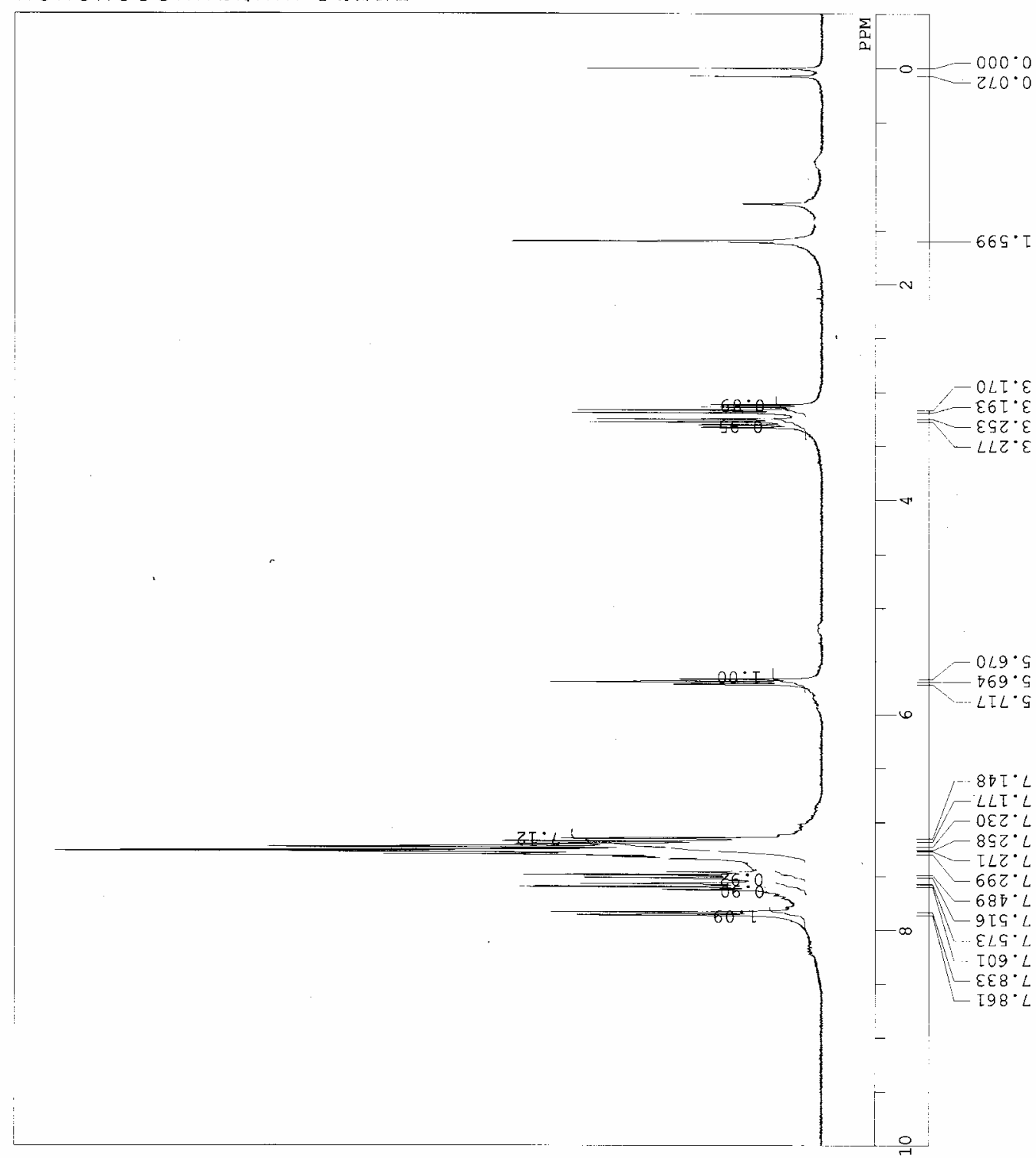




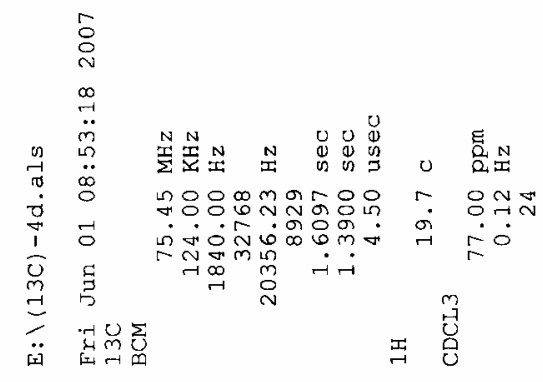

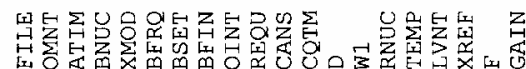

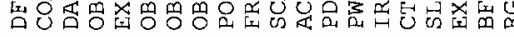

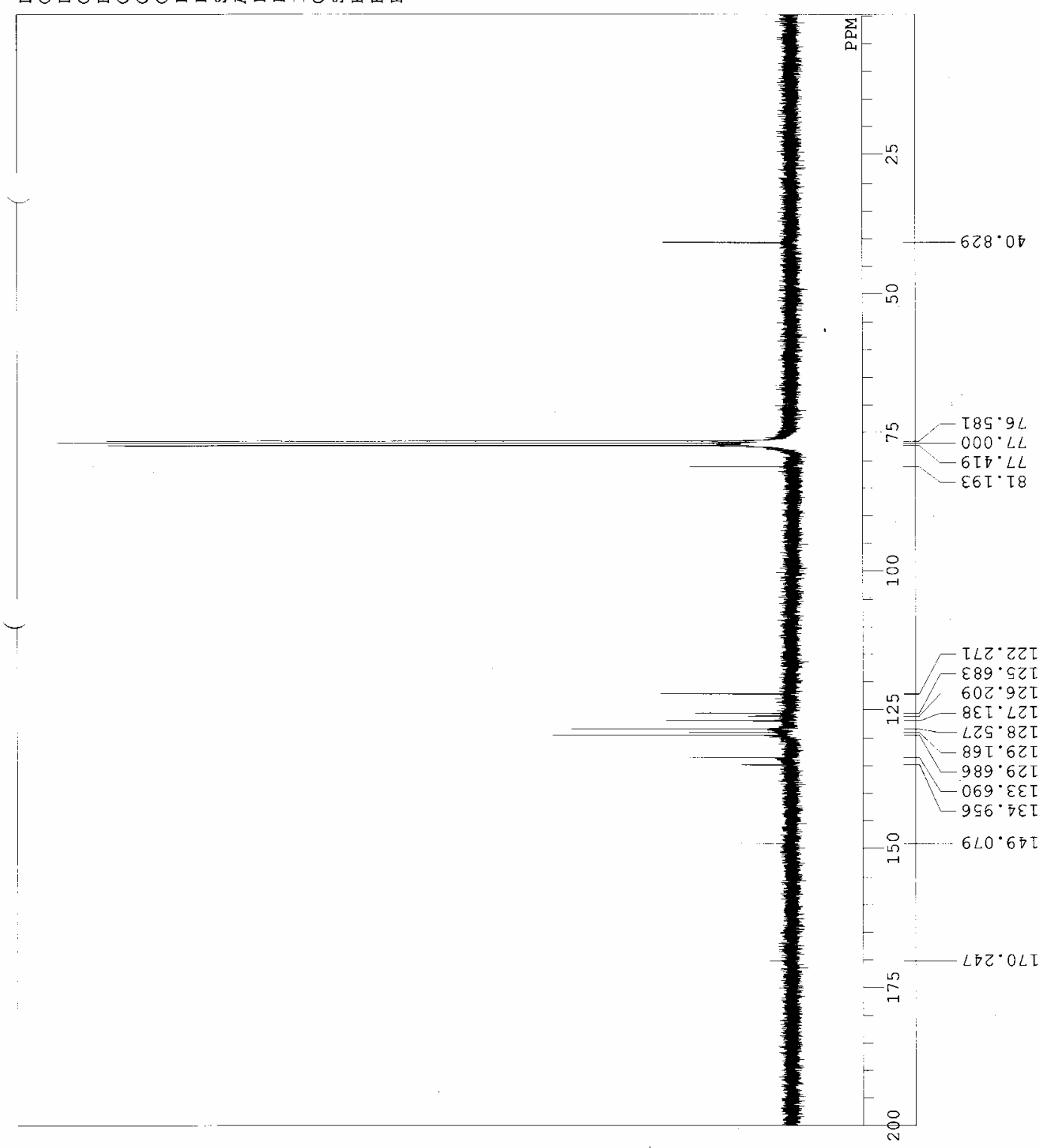




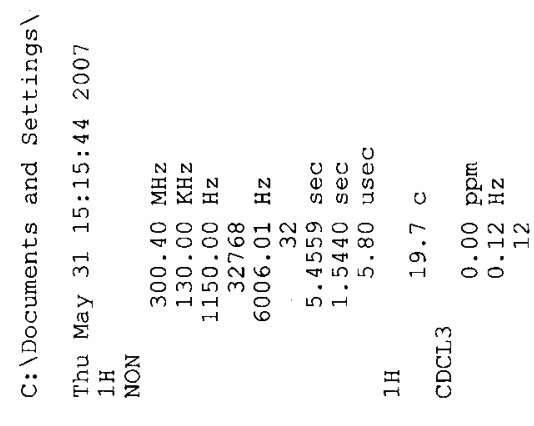

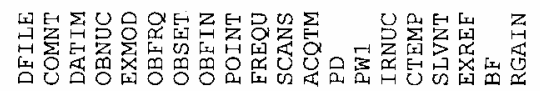

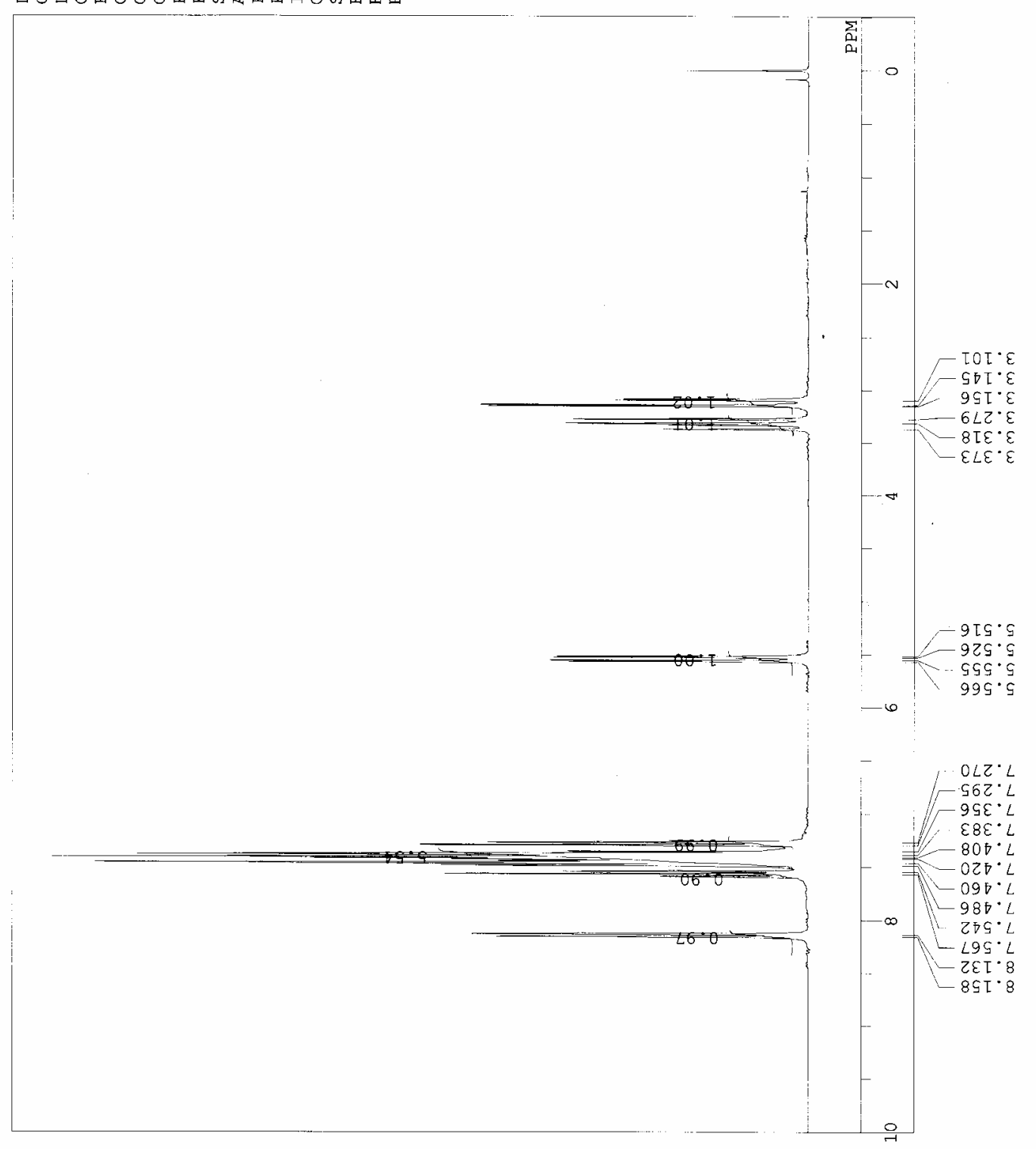




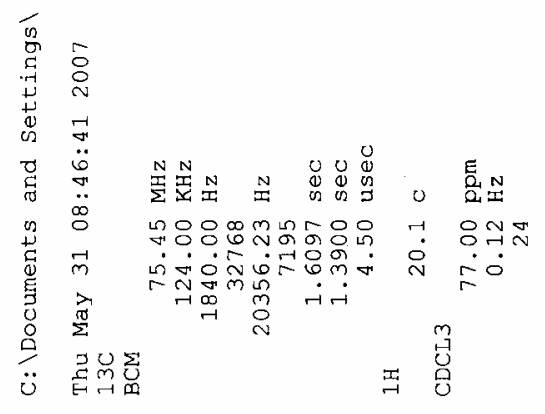

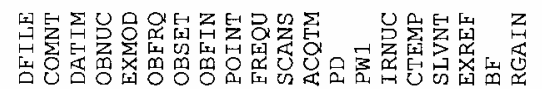

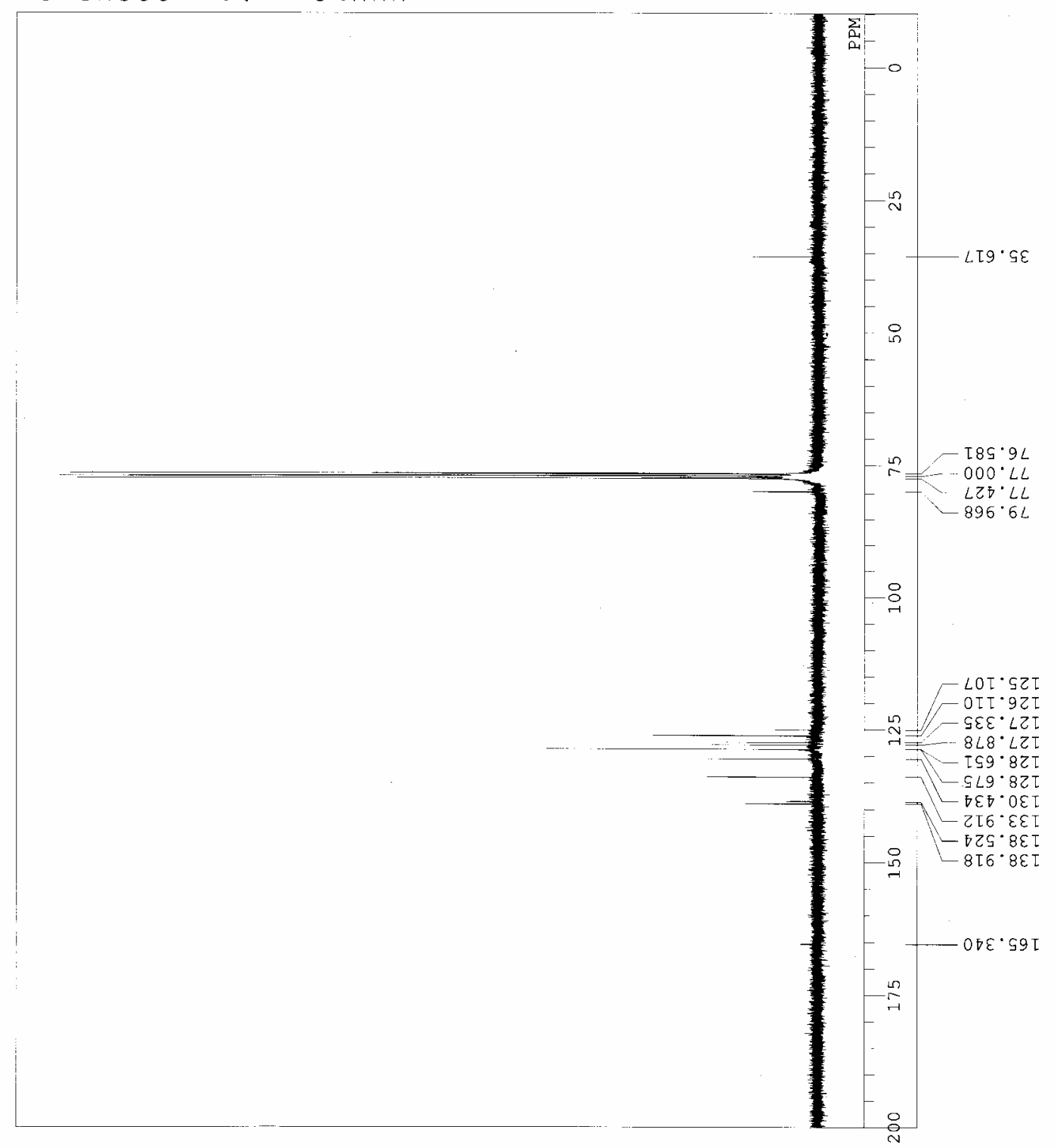

